\title{
NUCLEAR STAR FORMATION ACTIVITY AND BLACK HOLE ACCRETION IN NEARBY SEYFERT GALAXIES
}

\author{
P. Esquej ${ }^{1,2,3}$, A. Alonso-Herrero ${ }^{2,14}$, O. González-Martín ${ }^{4,5}$, S. F. Hönig ${ }^{6,7}$, A. Hernán-Caballero ${ }^{2,3}$, \\ P. Roche $^{8}$, C. Ramos Almeida ${ }^{4,5}$, R. E. Mason ${ }^{9}$, T. Díaz-Santos ${ }^{10}$, N. A. Levenson ${ }^{11}$, I. AretXaga ${ }^{12}$, \\ J. M. RodRíGUEZ EsPINOSA ${ }^{4,5}$, AND C. PACKHAM ${ }^{13}$ \\ ${ }^{1}$ Centro de Astrobiología, INTA-CSIC, Villafranca del Castillo, E-28850, Madrid, Spain \\ ${ }^{2}$ Instituto de Física de Cantabria, CSIC-Universidad de Cantabria, E-39005 Santander, Spain \\ ${ }^{3}$ Departamento de Física Moderna, Universidad de Cantabria, Avenida de Los Castros s/n, E-39005 Santander, Spain \\ ${ }^{4}$ Instituto de Astrofísica de Canarias (IAC), C/Vía Láctea, E-38205, La Laguna, Spain \\ ${ }^{5}$ Departamento de Astrofísica, Universidad de La Laguna (ULL), E-38205, La Laguna, Spain \\ ${ }^{6}$ UCSB Department of Physics, Broida Hall 2015H, Santa Barbara, CA, USA \\ ${ }^{7}$ Institut für Theoretische Physik und Astrophysik, Christian-Albrechts-Universität zu Kiel, Leibnizstrasse 15, D-24098 Kiel, Germany \\ ${ }^{8}$ Department of Physics, University of Oxford, Oxford OX1 3RH, UK \\ ${ }^{9}$ Gemini Observatory, Northern Operations Center, 670 North A'ohoku, HI 96720, USA \\ ${ }^{10}$ Spitzer Science Center, 1200 East California Boulevard, Pasadena, CA 91125, USA \\ ${ }^{11}$ Gemini Observatory, Casilla 603, La Serena, Chile \\ 12 Instituto Nacional de Astrofísica, Óptica y Electrónica (INAOE), Aptdo. Postal 51 y 216, 72000 Puebla, Mexico \\ ${ }^{13}$ Department of Physics and Astronomy, University of Texas at San Antonio, One UTSA Circle, San Antonio, TX 78249, USA \\ Received 2013 June 21; accepted 2013 November 3; published 2013 December 13
}

\begin{abstract}
Recent theoretical and observational works indicate the presence of a correlation between the star-formation rate (SFR) and active galactic nucleus (AGN) luminosity (and, therefore, the black hole accretion rate, $\dot{M}_{\mathrm{BH}}$ ) of Seyfert galaxies. This suggests a physical connection between the gas-forming stars on kpc scales and the gas on sub-pc scales that is feeding the black hole. We compiled the largest sample of Seyfert galaxies to date with high angular resolution ( 0.'4-0!.8) mid-infrared $(8-13 \mu \mathrm{m})$ spectroscopy. The sample includes 29 Seyfert galaxies drawn from the AGN Revised Shapley-Ames catalog. At a median distance of $33 \mathrm{Mpc}$, our data allow us to probe nuclear regions on scales of $\sim 65 \mathrm{pc}$ (median value). We found no general evidence of suppression of the $11.3 \mu \mathrm{m}$ polycyclic aromatic hydrocarbon (PAH) emission in the vicinity of these AGN, and we used this feature as a proxy for the SFR. We detected the $11.3 \mu \mathrm{m}$ PAH feature in the nuclear spectra of $45 \%$ of our sample. The derived nuclear SFRs are, on average, five times lower than those measured in circumnuclear regions of $600 \mathrm{pc}$ in size (median value). However, the projected nuclear SFR densities (median value of $22 M_{\odot} \mathrm{yr}^{-1} \mathrm{kpc}^{-2}$ ) are a factor of 20 higher than those measured on circumnuclear scales. This indicates that the SF activity per unit area in the central $\sim 65 \mathrm{pc}$ region of Seyfert galaxies is much higher than at larger distances from their nuclei. We studied the connection between the nuclear SFR and $\dot{M}_{\mathrm{BH}}$ and showed that numerical simulations reproduce our observed relation fairly well.
\end{abstract}

Key words: galaxies: nuclei - galaxies: Seyfert - infrared: galaxies

Online-only material: color figures

\section{INTRODUCTION}

One of the most important challenges in modern cosmology is to disentangle the physics behind the processes underlying galaxy formation and evolution. Observations over the past few decades have revealed that supermassive black holes (SMBHs) likely reside at the centers of all galaxies with a bulge, and that the properties of these black holes (BHs) and their host galaxies are tightly correlated (e.g., Magorrian et al. 1998; Ferrarese \& Merritt 2000; Gebhardt 2000; Kormendy \& Ho 2013). The coevolution of galaxies and their corresponding SMBHs depends on some physical mechanism, referred to as feedback, that links accretion and ejection of gas residing on a sub-pc scale in galactic nuclei to the rest of the galaxy (Silk \& Rees 1998; King 2010; Nayakshin \& Zubovas 2012). The connection between star formation (SF) activity on different physical scales in a galaxy and the presence of an active galactic nucleus (AGN) has been a long-discussed topic. However, there are still many uncertainties under consideration to disentangle

\footnotetext{
$\overline{{ }^{14} \text { Augusto G. Linares Senior Research Fellow. }}$
}

the processes behind such a relation (see, e.g., Hopkins \& Quataert 2010 and references therein).

In the standard unification model, the powering mechanism of AGN is gas accretion onto a central SMBH. However, the physics of angular momentum transfer to the vicinity of the BH is still unclear (see Alexander \& Hickox 2012 for a recent review). Given that the angular momentum of inflowing gas produced by galaxy mergers or other large scale structures (e.g., bars) cannot be removed instantaneously, many studies proposed that the inflowing gas could form a circumnuclear disk where SF can take place. Kawakatu \& Wada (2008 and references therein) put forward a model for such a circumnuclear disk, which might be coincident with the putative torus of the unification model of AGN (Antonucci 1993). This model predicts that SF would mostly take place in the outer parts of a $100 \mathrm{pc}-$ size torus (Wada \& Norman 2002). Cid Fernandes \& Terlevich (1995) proposed the presence of a starburst in the obscuring torus as a solution for the absence of conspicuous broad lines in Seyfert 2s. The starburst disk model of Thompson et al. (2005) estimates that most of the gas is supplied from outside the inner $200 \mathrm{pc}$, but this is better suited for ultraluminous 
infrared galaxies due to the high star-formation rates (SFRs) considered. Ballantyne (2008) presented an update of the Thompson et al. (2005) model with typical maximum SFRs of $\sim 1 M_{\odot} \mathrm{yr}^{-1}$ that could also potentially obscure the AGN. These nuclear pc-sized starbursts will mostly be associated with low luminosity AGN (i.e., Seyferts and low-ionization nuclear emission-line regions-LINERs). From an observational point of view, nuclear starbursts have been detected in Seyfert 2 galaxies and LINERs using UV images obtained with the Hubble Space Telescope (Heckman et al. 1995; González Delgado et al. 1998; Colina et al. 2002).

The numerical simulations of Hopkins \& Quataert (2010) predict a relation with some scatter between the SFR of the galaxy on different scales-going from $10 \mathrm{kpc}$ scale to the central parsec_-and the black hole accretion rate $\left(\dot{M}_{\mathrm{BH}}\right)$. This correlation appeared to be more prominent on smaller physical scales. However, simulations also indicate dynamical delays between the peaks of the SF and the BH growth (Hopkins 2012), in agreement with results from observational works (e.g., Davies et al. 2007; Wild et al. 2010; Ramos Almeida et al. 2013).

Mid-infrared (mid-IR) spectroscopy is a powerful tool to explore the nature of AGN and SF activity in galaxies. Among the most remarkable characteristics of the mid-IR spectra of galaxies is the presence of polycyclic aromatic hydrocarbon (PAH) emissions, with the most prominent features being at $6.2,7.7,8.6,11.3$, and $17 \mu \mathrm{m}$. They are due to the stretching and bending vibrations of aromatic hydrocarbon materials, where the shortest wavelength features are dominated by the smallest PAHs (e.g., Tielens 2010). This type of emission mostly originates in photo-dissociation regions where aromatic molecules are heated by the radiation field produced by young massive stars (Roche \& Aitken 1985; Roche et al. 1991). Therefore, PAHs are often used as indicators of the current SFR of galaxies. Note that they can also be excited by UV emission from B stars, and thus PAH emission probes SF over a few tens of million years (e.g., Peeters et al. 2004; Díaz-Santos et al. 2010).

PAH features are detected in AGN, although they generally appear weak when compared with those of star-forming galaxies (Roche et al. 1991). It has been proposed that the PAH molecules might be destroyed in the vicinity of an active nucleus due to the presence of a hard radiation field (Voit 1992a). There is also evidence that different PAHs might behave differently. Diamond-Stanic \& Rieke (2010) showed that the $11.3 \mu \mathrm{m}$ PAH feature emission is a reliable indicator of the SFR in AGN, at least for Seyfert-like AGN luminosities and kpc scales, while the $6.2,7.7$, and $8.6 \mu \mathrm{m}$ features appear suppressed. Signs of variations between the different features have been reported by many authors (e.g., Peeters et al. 2004; Galliano et al. 2008). For instance, Smith et al. (2007) found that the ratio of the PAH emission at 7.7 and $11.3 \mu \mathrm{m}$ is relatively constant among pure starbursts, while it decreases by up to a factor of five for galaxies hosting a weak AGN. They interpreted this as a selective destruction of the smallest PAH carriers by the hard radiation arising from the accretion disk, ruling out the explanation in terms of ionization of the molecules (see also Siebenmorgen et al. 2004).

A number of works have studied the SF activity using PAH emission and its relation to the AGN activity. Shi et al. (2007) demonstrated that the SF contribution increases from Palomar-Green QSO to 2MASS QSO and radio galaxies. Using measurements of the 7.7 and $11.3 \mu \mathrm{m}$ PAH using Spitzer/IRS data, they found higher SFRs for more intense nuclear activity, which indicates that the AGN selection technique influences the level of SF activity detected in the corresponding host galaxies. Watabe et al. (2008) investigated the nuclear versus circumnuclear SF for a sample of Seyfert galaxies using groundbased observations of the $3.3 \mu \mathrm{m}$ PAH feature. Assuming that the $3.3 \mu \mathrm{m}$ PAH traces the SF activity, they found that both SF and AGN activity are correlated (see also, Imanishi 2003; Imanishi \& Wada 2004). Such a relation implied that SF in the inner region of the AGN (within a few hundred parsecs from the center) might have a greater influence on $\dot{M}_{\mathrm{BH}}$. On the other hand, Mason et al. (2007) found weak or absent PAH emission in the central 20 pc of the Seyfert 1 galaxy NGC 1097, while in the circumnuclear region, strong 3.3 and $11.3 \mu \mathrm{m}$ PAH bands were detected. In the case of NGC 1097, the absence of PAH emission may be related to destruction/ionization of PAH molecules by hard photons from the nuclear star cluster.

Diamond-Stanic \& Rieke (2012) recently found a strong correlation between the kpc-scale SF derived using the $11.3 \mu \mathrm{m}$ PAH feature and $24 \mu \mathrm{m}$ observations for Seyfert galaxies. However, the limited angular resolution of their Spitzer data $\left(\sim 4^{\prime \prime}-5^{\prime \prime}\right)$ did not allow them to resolve nuclear $(\sim 100 \mathrm{pc})$ scales, and it is unclear if the measured PAH feature is associated with the galaxy or to the nuclear environment.

In this work we compile a sample of 29 Seyfert galaxies from the revised Shapley-Ames (RSA) galaxy catalog (Sandage \& Tammann 1987) with published ground-based mid-IR high angular resolution spectroscopy obtained on $8 \mathrm{~m}$-class telescopes. At a median distance of $33 \mathrm{Mpc}$, this sample allows us to study the nuclear SF activity around AGN on scales of $\sim 65 \mathrm{pc}$. We also use mid-IR spectra taken with the Infrared Spectrograph (IRS; Houck et al. 2004) on board Spitzer for all objects in our sample to investigate the extended ( $\sim 600 \mathrm{pc}) \mathrm{SF}$ in the host galaxy. This enables us to study the relation SFR-BHAR at different scales in the local universe.

This article is structured as follows: Section 2 describes the sample selection and data analysis. In Section 3, we study the nuclear $11.3 \mu \mathrm{m}$ PAH feature emission. Section 4 compares the circumnuclear and nuclear SF activity and its relation with $\dot{M}_{\mathrm{BH}}$. Finally, our conclusions are summarized in Section 5. Throughout this work we assumed a $\Lambda$ CDM cosmology with $\left(\Omega_{\mathrm{M}}, \Omega_{\Lambda}\right)=(0.3,0.7)$ and $H_{0}=70 \mathrm{~km} \mathrm{~s}^{-1} \mathrm{Mpc}^{-1}$.

\section{SAMPLE SELECTION AND DATA ANALYSIS}

\subsection{Sample}

Our sample (see Table 1) is drawn from the galaxymagnitude-limited RSA Seyfert sample, which includes the 89 Seyfert galaxies brighter than $B_{T}=13$ mag from Maiolino \& Rieke (1995) and Ho et al. (1997). We selected galaxies with existing high angular resolution $\left(\sim 00^{\prime} 4-0\right.$.' 8$)$ mid-IR spectra observed on $8 \mathrm{~m}$-class telescopes. The sample contains a total of 29 Seyfert galaxies, $16(55 \%)$ of which are Type 2 and $13(45 \%)$ of which are Type 1 AGN. We included in the Seyfert 1 category those galaxies classified as Seyfert 1.5, 1.8, and 1.9, as well as those with broad near-IR lines.

We used the hard 2-10 keV X-ray luminosity (see Table 1 for references) as a proxy for the AGN bolometric luminosities after correcting for absorption and applying the bolometric corrections of Marconi et al. (2004). The high column density in Compton-thick objects (defined as those having $N_{\mathrm{H}}>$ $10^{24} \mathrm{~cm}^{-2}$, see Table 1) prevents us from measuring the intrinsic nuclear luminosity below $10 \mathrm{keV}$, instead one can only derive the reflection component from model fitting. Assuming that the 
Table 1

Sample Properties

\begin{tabular}{|c|c|c|c|c|c|c|c|}
\hline Object & $\begin{array}{c}D_{L}^{\mathrm{a}} \\
(\mathrm{Mpc})\end{array}$ & $b / a$ & Type & $\begin{array}{c}\log L_{2-10 \mathrm{keV}^{\mathrm{b}}} \\
\left(\mathrm{erg} \mathrm{s}^{-1}\right)\end{array}$ & $\begin{array}{l}\log L_{\mathrm{agn}}{ }^{\mathrm{c}} \\
\left(\mathrm{erg} \mathrm{s}^{-1}\right)\end{array}$ & $\begin{array}{c}\log \left(M_{\mathrm{BH}}\right) \\
\left(M_{\odot}\right)\end{array}$ & Refs. \\
\hline Circinus & 4.2 & 0.4 & Sy2 & $42.6^{*}$ & 43.8 & 6.42 & 1 \\
\hline ESO 323-G077 & 65.0 & 0.7 & Sy1 & 42.7 & 43.9 & 7.40 & 2 \\
\hline IC 5063 & 49.0 & 0.7 & Sy2 & 42.8 & 44.0 & 7.74 & 1 \\
\hline Mrk 509 & 151.2 & 0.8 & Sy1 & 43.9 & 45.4 & 7.86 & $3,4,5$ \\
\hline NGC 1068 & 16.3 & 0.9 & Sy2 & $43.0^{*}$ & 44.3 & 7.59 & 1 \\
\hline NGC 1365 & 23.5 & 0.5 & Sy1 & $42.1^{\mathrm{d}}$ & 43.1 & 8.20 & 6 \\
\hline NGC 1386 & 12.4 & 0.4 & Sy2 & $41.6^{*}$ & 42.6 & 7.42 & 1 \\
\hline NGC 1808 & 14.3 & 0.6 & Sy2 & 40.4 & 41.2 & $\ldots$ & 7 \\
\hline NGC 2110 & 33.6 & 0.7 & Sy1 & 42.6 & 43.7 & 8.30 & $3,4,8$ \\
\hline NGC 2992 & 33.2 & 0.3 & Sy1 & 43.1 & 44.4 & 7.72 & 8 \\
\hline NGC 3081 & 34.4 & 0.9 & Sy2 & 42.5 & 43.6 & 7.13 & 1 \\
\hline NGC 3227 & 16.6 & 0.7 & Sy1 & 42.4 & 43.5 & 7.62 & $4,9,10$ \\
\hline NGC 3281 & 46.1 & 0.5 & Sy2 & 42.6 & 43.8 & 7.91 & 1 \\
\hline NGC 3783 & 42.0 & 0.9 & Sy1 & 43.2 & 44.5 & 7.48 & $3,4,10$ \\
\hline NGC 4151 & 14.3 & 0.7 & Sy1 & 42.1 & 43.2 & 7.66 & 11,12 \\
\hline NGC 4388 & 36.3 & 0.2 & Sy2 & 42.9 & 44.1 & 7.23 & 1 \\
\hline NGC 4507 & 51.0 & 0.8 & Sy2 & 43.1 & 44.4 & 7.65 & 1 \\
\hline NGC 4945 & 3.6 & 0.2 & Sy2 & $42.3^{*}$ & 43.4 & 6.15 & 13,14 \\
\hline NGC 5128 & 3.7 & 0.8 & Sy2 & 41.9 & 42.9 & 7.84 & 4 \\
\hline NGC 5135 & 59.3 & 0.7 & Sy 2 & $43.1^{*}$ & 44.4 & 7.29 & 1 \\
\hline NGC 5347 & 33.6 & 0.8 & Sy2 & $42.4^{*}$ & 43.5 & 6.97 & 1,15 \\
\hline NGC 5506 & 26.6 & 0.3 & Sy1 & 43.0 & 44.3 & 7.95 & 1 \\
\hline NGC 5643 & 17.2 & 0.9 & Sy2 & 41.4 & 42.3 & 7.40 & 16,15 \\
\hline NGC 7130 & 70.0 & 0.9 & Sy2 & $43.1^{*}$ & 44.4 & 7.59 & 1 \\
\hline NGC 7172 & 37.4 & 0.6 & Sy2 & 42.2 & 43.3 & 7.67 & 17 \\
\hline NGC 7213 & 25.1 & 0.9 & Sy1 & 42.1 & 43.1 & 7.74 & 5 \\
\hline NGC 7469 & 70.8 & 0.7 & Sy1 & 43.3 & 44.7 & 7.08 & $3,4,6$ \\
\hline NGC 7479 & 34.2 & 0.7 & Sy1 & 42.0 & 43.0 & 7.68 & 7,10 \\
\hline NGC 7582 & 22.6 & 0.4 & Sy1 & $41.9^{\mathrm{d}}$ & 42.9 & 7.13 & 1,18 \\
\hline
\end{tabular}

Notes.

a Distances from NED.

${ }^{\text {b }}$ Compton-thick sources according to Marinucci et al. (2012). Hard X-ray luminosities are corrected by a factor of 70.

c AGN bolometric luminosities calculated from X-ray luminosities after applying the bolometric corrections of Marconi et al. (2004).

${ }^{\mathrm{d}}$ Changing-look AGN (e.g., Bianchi et al. 2005). Data from an intermediate state.

* Compton-thick sources.

References. (1) Marinucci et al. 2012; (2) Malizia et al. 2007; (3) Dadina 2007; (4) Tueller et al. 2008; (5) Asmus et al. 2011; (6) Risaliti et al. 2005; (7) Brightman \& Nandra 2011; (8) Woo \& Urry 2002; (9) Hönig et al. 2010; (10) Diamond-Stanic \& Rieke 2012; (11) Wang et al. 2011; (12) Beckmann et al. 2006; (13) Guainazzi et al. 2000; (14) Mueller et al. 2003; (15) Beifiori et al. 2009; (16) Guainazzi et al. 2004; (17) Akylas et al. 2001; (18) Piconcelli et al. 2007.

[O III] forbidden line is a tracer of the AGN intrinsic luminosity and comparing it with the observed hard X-ray emission of Compton-thick AGN, Marinucci et al. (2012) derived a correction factor of 70 , which we used to correct the observed $2-10 \mathrm{keV}$ luminosities of these objects. ${ }^{15}$ This large correction factor is also theoretically justified by the torus model proposed by Ghisellini et al. (1994). This is the method commonly used for Compton thick sources and applied in other several works, as in, e.g., Bassani et al. (1999) and Panessa et al. (2006). The nuclear Mid-IR and X-ray luminosities are well correlated (e.g., Levenson et al. 2009; Asmus et al. 2011), which is also fulfilled for sources in our sample (Hönig et al. 2010; GonzálezMartín et al. 2013). The only significant outlier, NGC 1808, indicates that for this source the AGN does not dominate the continuum mid-IR emission (see Figure 5 in González-Martín et al. 2013). The uncertainties in $L_{\mathrm{agn}}$ are driven by the scatter on

\footnotetext{
15 The X-ray luminosity of NGC 7479 is not corrected by such a factor because, despite its high $N_{\mathrm{H}}$, its Compton-thick nature is not confirmed. Therefore, its luminosity could be up to a factor of 60-70 times higher (Panessa et al. 2006).
}

the relationship for the bolometric correction which, in general, is significantly larger than the error on the X-ray luminosities. Based on the $L_{\text {agn }}$ determination, Young et al. (2010) derived typical uncertainties of 0.4 dex (see also Marinucci et al. 2012).

Our sample spans AGN bolometric luminosities in the range of $\log L_{\text {agn }}=41.2-45.5 \mathrm{erg} \mathrm{s}^{-1}$, with a median value of $43.7 \mathrm{erg} \mathrm{s}^{-1}$. This is a fair representation of the full RSA Seyfert sample (see Figure 1 of Diamond-Stanic \& Rieke 2012). ${ }^{16}$ As can be seen from Figure 1, Type 1 and Type 2 sources have similar distributions of $L_{\mathrm{agn}}$, with median values (in logarithm scale) of 43.7 and $43.8 \mathrm{erg} \mathrm{s}^{-1}$ for Sy1 and Sy2s, respectively. We also list in Table 1 the BH masses of the galaxies in our sample and corresponding references. There is no available $\mathrm{BH}$ mass measurement for NGC 1808. The median value for our sample is $3.9 \times 10^{7} M_{\odot}$, which is similar to the $3.2 \times 10^{7} M_{\odot}$ median value for the RSA Seyfert sample (Diamond-Stanic \& Rieke 2012). In terms of the Eddington ratio, we sample values of $L_{\text {agn }} / L_{\text {edd }}=10^{-4}-0.3$.

\footnotetext{
${ }^{16}$ Note that in this figure they used the luminosity of the mid-IR line [O IV] as the proxy for the AGN bolometric luminosities.
} 


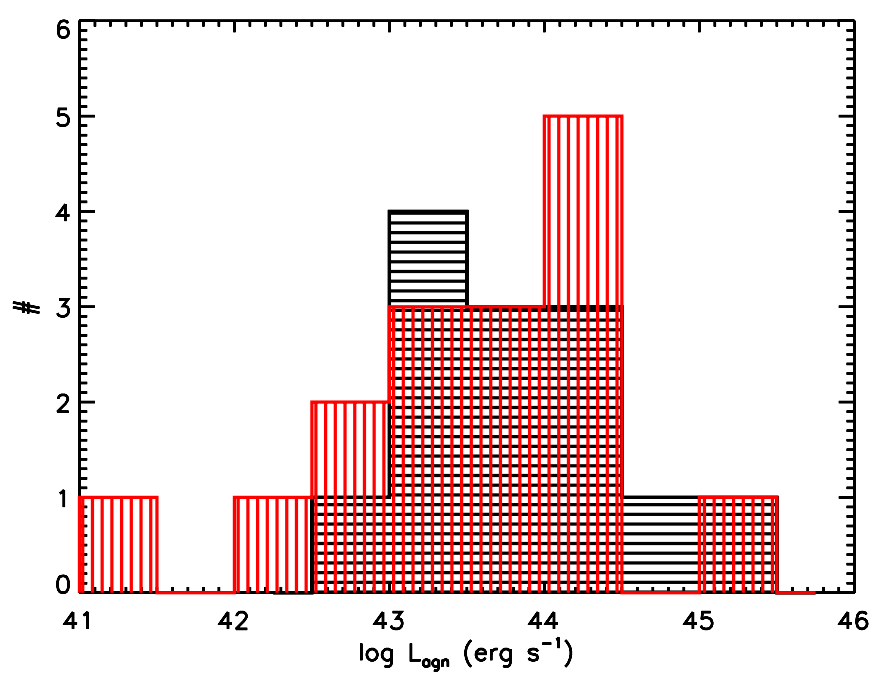

Figure 1. Distribution of the AGN bolometric luminosities for Sy1 (black histogram, horizontal filling lines) and Sy2 (red histogram, vertical lines) galaxies in our sample.

(A color version of this figure is available in the online journal.)

\subsection{Observations}

Ground-based mid-IR spectroscopic observations of the 29 Seyfert galaxies were taken with three different instruments. They operate on $8 \mathrm{~m}$-class telescopes and cover the $N$ band, $\sim 8-13 \mu \mathrm{m}$. Table 2 summarizes details of the midIR spectroscopic data along with the references where the observations where originally published. The observations taken with the Thermal-Region Camera Spectrograph (T-ReCS; Telesco et al. 1998) on the $8.1 \mathrm{~m}$ Gemini-South Telescope used the low resolution mode, which provides a spectral resolution of $R=\Delta \lambda / \lambda \sim 100$, and slit widths between 0 '.31 and 0.70 . The observations performed by Michelle (Glasse et al. 1997) on the $8.1 \mathrm{~m}$ Gemini-North telescope, which has a higher spectral resolution by a factor of two $(R \sim 200)$, were obtained with slit widths of $\sim 0$ '.4. Finally, observations with the Very Large Telescope (VLT) spectrometer and imager for the mid-infrared (VISIR; Lagage et al. 2004) instrument mounted on the $8.2 \mathrm{~m}$ VLT UT3 telescope at the ESO/Paranal observatory were obtained with the low spectral resolution mode $(R \sim 300)$ and a slit width of 0 '.75 or $1^{\prime \prime}$ (and 0.'4 for NGC 1068). For the typical distances of our sample the ground-based slit width probe typical physical scales of $\sim 65 \mathrm{pc}$. These range from $\sim 7-255 \mathrm{pc}$ for all the objects except for Mrk 509 (545 pc), which is by far the most distant galaxy in the sample.

Sixteen sources were observed with Gemini/T-ReCS (González-Martín et al. 2013 and references therein). Thirteen sources have VLT/VISIR observations (see Hönig et al. 2010, for details), with 4 overlapping with the T-ReCS sample. Finally, six Seyfert galaxies were observed with Gemini/Michelle (Mason et al. 2006; Alonso-Herrero et al. 2011; Colling 2011), of which two sources also have VISIR observations. We refer the reader to the original articles for details on the observations and the data reduction.

We retrieved mid-IR Spitzer/IRS spectra (for all sources except for NGC 1068) from the Cornell Atlas of Spitzer/ IRS Source (CASSIS v4; Lebouteiller et al. 2011). We used staring mode observations taken with the short-low (SL) module covering the spectral range $\sim 5-15 \mu \mathrm{m}$. The spectral resolution is $R \sim 60-120$. The CASSIS database provides spectra with optimal extraction regions to ensure the best signal-to-noise
Table 2

Ground-Based High Angular Resolution Mid-IR Spectroscopy

\begin{tabular}{|c|c|c|c|}
\hline Galaxy & Instrument & $\begin{array}{l}\text { Slit } \\
\left({ }^{\prime \prime}\right)\end{array}$ & Refs \\
\hline ESO 323-G077 & VISIR & 0.75 & 4 \\
\hline \multirow[t]{2}{*}{ IC 5063} & T-ReCS & 0.67 & 2,5 \\
\hline & VISIR & 1.00 & 4 \\
\hline Mrk 509 & VISIR & 0.75 & 4 \\
\hline \multirow[t]{2}{*}{ NGC 1068} & Michelle & 0.36 & 6 \\
\hline & VISIR & 0.40 & 4 \\
\hline NGC 1365 & T-ReCS & 0.35 & 2,7 \\
\hline NGC 1386 & T-ReCS & 0.31 & 2 \\
\hline NGC 1808 & T-ReCS & 0.35 & 2,8 \\
\hline \multirow[t]{2}{*}{ NGC 2110} & Michelle & 0.36 & 9 \\
\hline & VISIR & 0.75 & 4 \\
\hline NGC 2992 & Michelle & 0.40 & 10 \\
\hline NGC 3081 & T-ReCS & 0.65 & 2 \\
\hline NGC 3281 & T-ReCS & 0.35 & 2,11 \\
\hline NGC 3227 & VISIR & 0.75 & 4 \\
\hline NGC 3783 & VISIR & 0.75 & 4 \\
\hline NGC 4151 & Michelle & 0.36 & 12 \\
\hline NGC 4388 & Michelle & 0.40 & 10 \\
\hline NGC 4507 & VISIR & 1.00 & 4 \\
\hline NGC 5135 & T-ReCS & 0.70 & 2,13 \\
\hline NGC 5347 & Michelle & 0.40 & 10 \\
\hline NGC 5506 & $\mathrm{~T}-\mathrm{ReCS}$ & 0.36 & 2,14 \\
\hline \multirow[t]{2}{*}{ NGC 5643} & T-ReCS & 0.36 & 2 \\
\hline & VISIR & 0.75 & 4 \\
\hline NGC 7130 & T-ReCS & 0.70 & 2,13 \\
\hline NGC 7172 & T-ReCS & 0.36 & 2,14 \\
\hline NGC 7213 & VISIR & 0.75 & 4 \\
\hline NGC 7469 & VISIR & 0.75 & 4 \\
\hline NGC 7479 & T-ReCS & 0.35 & 2 \\
\hline \multirow[t]{2}{*}{ NGC 7582} & T-ReCS & 0.70 & 2 \\
\hline & VISIR & 0.75 & 3 \\
\hline
\end{tabular}

References. (1) Roche et al. 2006; (2) González-Martín et al. 2013; (3) Hönig et al. 2008; (4) Hönig et al. 2010; (5) Young et al. 2007; (6) Mason et al. 2006; (7) Alonso-Herrero et al. 2012; (8) Sales et al. 2013; (9) Mason et al. 2009; (10) Colling 2011; (11) Sales et al. 2011; (12) Alonso-Herrero et al. 2011; (13) Díaz-Santos et al. 2010; (14) Roche et al. 2007.

ratio and are fully reduced. We only needed to apply a small offset to stitch together the two short-wavelength modules SL1 and SL2 (but note that this does not affect the PAH measurement, see Section 2.3). NGC 1068 is part of the GOALS programme (Armus et al. 2009), and IRS SH data have been obtained from the NASA/IPAC Infrared Science Archive. Assuming a typical spatial resolution of $3^{\prime \prime} .7$ for the SL module of IRS given by the slit width, this corresponds to a physical scale of about $\sim 600$ pc for our sample, i.e., a factor of 10 less resolved than for the ground-based data. Figure 2 shows a comparison of Spitzer against ground-based data of NGC 7130 for illustration. We present the spectra of the full sample in Figure 8 in the Appendix.

\subsection{Measuring the $11.3 \mu \mathrm{m}$ PAH Feature}

A number of methods have been developed to accurately measure the fluxes of PAH features, in particular for the relatively large spectral range covered by IRS. These include, among others, PAHFIT (Smith et al. 2007), DecompIR (Mullaney et al. 2011), and spline fit (e.g., Uchida et al. 2000; Peeters et al. 2002). They are useful for decomposing IR spectra, especially when the AGN emission is contaminated by extranuclear emission. These techniques, however, might not be appropriate 


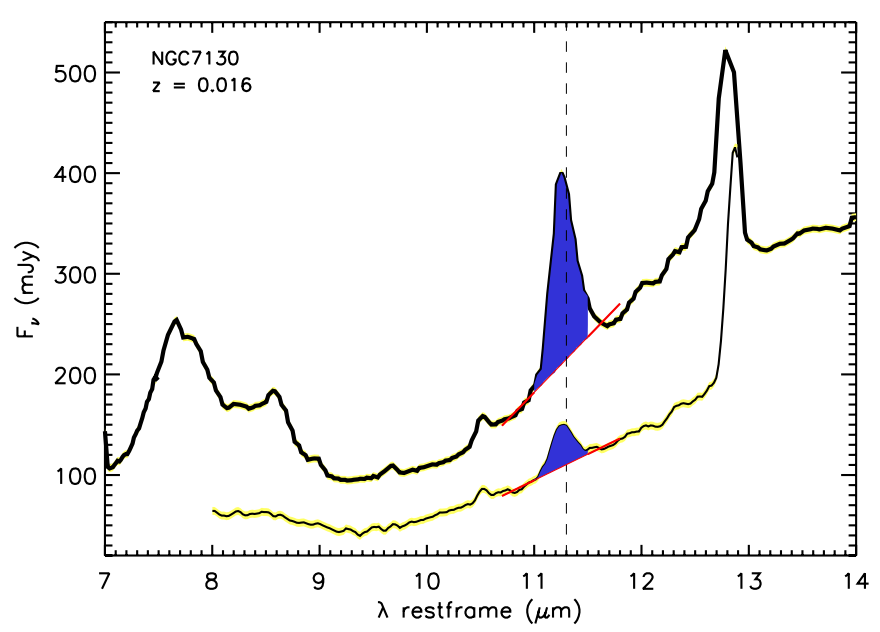

Figure 2. Spitzer/IRS SL spectrum (thick line) from CASSIS compared with the ground-based nuclear T-ReCS spectrum (thin line) from González-Martín et al. (2013) of NGC 7130, one of the galaxies in our sample. We show the location of the $11.3 \mu \mathrm{m}$ PAH feature, with the blue shaded area indicating the spectral range used for obtaining the integrated flux. The red lines are the fitted local continua. The rms of the spectrum is shown in yellow. We note that the [Ne II] $12.81 \mu \mathrm{m}$ emission line is contaminated by the $12.7 \mu \mathrm{m}$ PAH feature, which cannot be resolved. The complete sample is shown in Figure 8 in the Appendix

(A color version of this figure is available in the online journal.)

for the limited wavelength coverage of ground-based data and/or weak PAH features (see Smith et al. 2007).

We measured the flux of the $11.3 \mu \mathrm{m}$ PAH feature following the method described in Hernán-Caballero \& Hatziminaoglou (2011). We fitted a local continuum by linear interpolation of the average flux in two narrow bands on both sides of the PAH. We then subtracted this fitted local continuum and integrated the residual data in a spectral range centered on $11.3 \mu \mathrm{m}\left(\lambda_{\text {rest }}=\right.$ $11.05-11.55 \mu \mathrm{m})$, to obtain the PAH flux. Figure 2 illustrates the method. As can be seen from this figure, this procedure slightly underestimates the PAH feature flux due to losses at the wings of the line profiles and overlaps between adjacent $\mathrm{PAH}$ bands. We corrected for these effects by assuming that the line has a Lorentzian profile of known width and by applying a multiplicative factor (see Hernán-Caballero \& Hatziminaoglou 2011 for details). We measured the equivalent width (EW) of the $11.3 \mu \mathrm{m}$ PAH feature by dividing the integrated PAH flux by the interpolated continuum at the center of the feature. We derived the uncertainties in the measurements by performing Monte Carlo simulations. This was done by calculating the dispersion around the measured fluxes and EWs in a hundred simulations of the original spectrum with random noise distributed as the rms.

Additionally, PAH fluxes measured using a local continuum tend to be smaller than those using a continuum fitted over a large spectral range. To scale up our flux values to the total emission in the PAH features, we used the multiplicative factor of two. This value was derived by Smith et al. (2007) for the $11.3 \mu \mathrm{m}$ PAH, after comparing results obtained by the spline fitting and the PAHFIT full decomposition. For consistency in all measurements, we used the same method described above for both the ground-based and IRS spectra. We detected the $11.3 \mu \mathrm{m}$ $\mathrm{PAH}$ feature at the $2 \sigma$ level or higher significance in all objects of our sample observed with IRS, except for NGC 3783 (see Figures 2 and 8). Our measurements of the $11.3 \mu \mathrm{m}$ PAH fluxes agree well with those of Diamond-Stanic \& Rieke (2012) using PAHFIT, even though they used their own spectral extraction from IRS data.

\section{NUCLEAR $11.3 \mu \mathrm{m}$ PAH FEATURE EMISSION}

In this section, we investigate the nuclear $11.3 \mu \mathrm{m}$ PAH feature emission in our sample of galaxies. Hereinafter, nuclear scales generally refer to the physical regions observed with the T-ReCS/Michelle/VISIR instruments, whereas circumnuclear scales are those probed with the IRS spectroscopy. The only exceptions are the most nearby (distances of $\sim 4 \mathrm{Mpc}$ ) galaxies Circinus, NGC 4945, and NGC 5128. To explore similar physical regions in comparison to the rest of the sample we used the IRS observations as our nuclear spectra for the three galaxies. For these sources the circumnuclear data are from Siebenmorgen et al. (2004) and Galliano et al. (2008; see Table 3 for more information).

The median value of the nuclear physical sizes probed with our data is $65 \mathrm{pc}$ (see Table 3). If two nuclear spectra exist for the same galaxy, we used the one sampling that was of a physical scale closest to the median value for consistency with the rest of the galaxies. This information along with the physical scales probed with the nuclear spectra are given in Table 3 . Note that this is approximately a factor of 10 improvement in physical resolution with respect to the circumnuclear median value of $600 \mathrm{pc}$. Hereinafter, we will use the term size as referring to the physical scale probed, which is determined by the slit widths of the observations.

\subsection{Detection of the 11.3 $\mathrm{m}$ PAH Feature}

The $11.3 \mu \mathrm{m}$ PAH feature is weak or it might not even be present in a large fraction of galaxies in our sample, as can be seen from the nuclear spectra in Figures 2 and 8 . We deemed the feature as detected if the integrated flux is, at least, two times above the corresponding measured error. This is equivalent to having the PAH feature detected with a significance of $2 \sigma$ or higher. The nondetections are given as upper limits at a $2 \sigma$ level, that is, with a $95 \%$ probability that the real flux is below the quoted value.

Table 3 gives the nuclear luminosities and EWs of the $11.3 \mu \mathrm{m}$ PAH detections, as well as the $2 \sigma$ upper limits for the remaining objects. Note that the flux of the $11.3 \mu \mathrm{m}$ PAH feature is not corrected for extinction. Thus, its proper characterization might be hampered in cases of high extinction, i.e., when the PAH molecules are embedded behind the silicate grains and the feature is buried within the silicate absorption at $\sim 9.7 \mu \mathrm{m}$. This also depends on the location of the material causing the extinction relative to the $\mathrm{PAH}$ emitting region. Another additional complication is the presence of crystalline silicate absorption at around $11 \mu \mathrm{m}$, which has been detected in local ultraluminous infrared galaxies (Spoon et al. 2006) and in local Seyferts (Roche et al. 2007). In particular, Colling (2011) detected crystalline silicate absorption that could be blended with the $11.3 \mu \mathrm{m}$ PAH feature in some of the galaxies in our sample, namely, NGC 4388, NGC 5506, NGC 7172, and NGC 7479 (see also Section 3.2).

Using the T-ReCS/VISIR/Michelle data we detected nuclear $11.3 \mu \mathrm{m}$ PAH emission in 10 galaxies. For the three most nearby Seyferts the $11.3 \mu \mathrm{m}$ PAH feature is detected in the IRS observations, while in the corresponding T-ReCS/VISIR spectra the feature is below the detection limits. Taking this into account, we detected nuclear $11.3 \mu \mathrm{m}$ PAH feature emission in 13 out of 29 galaxies ( $45 \%$ of the sample). The detection rate is similar for Seyfert 1s and Seyfert 2s (40\% and 50\%, respectively). The observed EWs of the feature (using the fitted 
Table 3

Nuclear Measurements of the Sample

\begin{tabular}{|c|c|c|c|c|c|c|c|}
\hline Name & Ins & $\begin{array}{l}\text { Slit/Size } \\
\left({ }^{\prime \prime} / \mathrm{pc}\right)\end{array}$ & $\begin{array}{c}L_{11.3 \mu \mathrm{mPAH}} \\
\left(10^{40} \mathrm{erg} \mathrm{s}^{-1}\right)\end{array}$ & $\begin{array}{c}\mathrm{EW}_{11.3 \mu \mathrm{m} \text { PAH }} \\
\left(10^{-3} \mu \mathrm{m}\right)\end{array}$ & $\begin{array}{l}\text { SFR } \text { nuclear }_{\text {no }} \\
\left(M_{\odot} \mathrm{yr}^{-1}\right)\end{array}$ & $\mathrm{SFR}_{\text {nuclear }} / \mathrm{SFR}_{\text {circ }}$ & $\left(f_{11.3 \mu \mathrm{mPAH}}\right) /\left(f_{[\mathrm{Ne} \mathrm{II}]}\right)$ \\
\hline Circinus $^{\mathrm{a}}$ & IRS & $3.70 / 75$ & $5.1 \pm 0.3$ & $61 \pm 1$ & 0.13 & 0.23 & 0.83 \\
\hline ESO323-G077 & VISIR & $0.75 / 235$ & $<9.3$ & $<9$ & $<0.23$ & $<0.07$ & $<0.56$ \\
\hline IC5063 & T-ReCS & $0.65 / 153$ & $<8.4$ & $<12$ & $<0.21$ & $<0.53$ & $\ldots$ \\
\hline Mrk509 & VISIR & $0.75 / 545$ & $44.5 \pm 17.6$ & $10 \pm 4$ & 1.11 & 0.18 & $\ldots$ \\
\hline NGC 1068 & Michelle & $0.36 / 28$ & $14.4 \pm 2.7$ & $9 \pm 1$ & 0.36 & 0.36 & $\ldots$ \\
\hline NGC 1365 & T-ReCS & $0.35 / 40$ & $<1.9$ & $<18$ & $<0.05$ & $<0.06$ & $<0.97$ \\
\hline NGC 1386 & T-ReCS & $0.31 / 19$ & $<0.5$ & $<31$ & $<0.01$ & $<0.26$ & $<0.91$ \\
\hline NGC 1808 & T-ReCS & $0.35 / 24$ & $8.2 \pm 0.5$ & $365 \pm 22$ & 0.21 & 0.17 & 0.74 \\
\hline NGC 2110 & VISIR & $0.75 / 121$ & $<1.3$ & $<7$ & $<0.03$ & $<0.12$ & $\ldots$ \\
\hline NGC 2992 & Michelle & $0.40 / 64$ & $<3.8$ & $<30$ & $<0.09$ & $<0.14$ & $<0.33$ \\
\hline NGC 3081 & T-ReCS & $0.65 / 107$ & $<1.9$ & $<18$ & $<0.05$ & $<0.25$ & $<0.34$ \\
\hline NGC 3227 & VISIR & $0.75 / 60$ & $2.9 \pm 0.5$ & $63 \pm 11$ & 0.07 & 0.18 & 0.58 \\
\hline NGC 3281 & T-ReCS & $0.35 / 78$ & $<6.2$ & $<9$ & $<0.16$ & $<0.98$ & $<0.40$ \\
\hline NGC 3783 & VISIR & $0.75 / 151$ & $<3.3$ & $<6$ & $<0.08$ & $<0.79$ & $<0.22$ \\
\hline NGC 4151 & Michelle & $0.36 / 25$ & $<2.4$ & $<17$ & $<0.06$ & $<1.03$ & $<0.36$ \\
\hline NGC 4388 & Michelle & $0.40 / 70$ & $<7.7$ & $<68$ & $<0.19$ & $<0.38$ & $<0.18$ \\
\hline NGC 4507 & VISIR & $1.00 / 245$ & $<4.5$ & $<5$ & $<0.11$ & $<0.12$ & $<0.10$ \\
\hline NGC $4945^{\mathrm{b}}$ & IRS & $3.70 / 64$ & $0.4 \pm 0.1$ & $358 \pm 17$ & 0.01 & 0.13 & 0.13 \\
\hline NGC $5128^{a}$ & IRS & $3.70 / 66$ & $0.6 \pm 0.1$ & $65 \pm 2$ & 0.01 & 0.18 & 0.43 \\
\hline NGC $5135^{\mathrm{c}}$ & T-ReCS & $0.70 / 200$ & $5.9 \pm 2.2$ & $34 \pm 12$ & 0.15 & 0.04 & 0.63 \\
\hline NGC 5347 & Michelle & $0.40 / 65$ & $<6.5$ & $<56$ & $<0.16$ & $<0.76$ & $<0.64$ \\
\hline NGC 5506 & T-ReCS & $0.35 / 45$ & $6.4 \pm 2.8$ & $15 \pm 6$ & 0.16 & 0.32 & 0.40 \\
\hline NGC 5643 & VISIR & $0.75 / 62$ & $1.6 \pm 0.2$ & $49 \pm 4$ & 0.04 & 0.24 & 0.29 \\
\hline NGC $7130^{c}$ & T-ReCS & $0.70 / 236$ & $47.1 \pm 3.3$ & $166 \pm 11$ & 1.18 & 0.22 & 1.11 \\
\hline NGC 7172 & T-ReCS & $0.35 / 63$ & $<2.8$ & $<43$ & $<0.07$ & $<0.16$ & $<0.27$ \\
\hline NGC 7213 & VISIR & $0.75 / 91$ & $<0.7$ & $<8$ & $<0.02$ & $<0.14$ & $<0.06$ \\
\hline NGC 7469 & VISIR & $0.75 / 255$ & $47.7 \pm 4.7$ & $31 \pm 3$ & 1.19 & 0.10 & 0.82 \\
\hline NGC 7479 & T-ReCS & $0.35 / 58$ & $<5.6$ & $<37$ & $<0.14$ & $<0.82$ & $\ldots$ \\
\hline NGC 7582 & T-ReCS & $0.70 / 76$ & $3.9 \pm 0.7$ & $50 \pm 9$ & 0.10 & 0.30 & 0.41 \\
\hline
\end{tabular}

Notes. The 11.3 $\mu \mathrm{m}$ PAH luminosities include a multiplicative factor of two for comparison with PAHFIT measurements (see Section 2.3). The values of the EW and $f_{11.3 \mu \mathrm{mPAH}} / f_{[\mathrm{Ne} \mathrm{II}]}$ are from measurements using the fitted local continuum. Upper limits at a $2 \sigma$ significance are included for nondetections with the $<$ symbol. Circumnuclear SFR are from Infrared Space Observatory $11.3 \mu \mathrm{m}$ PAH measurements

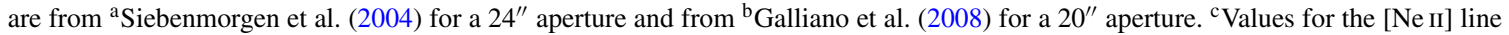
are from Díaz-Santos et al. (2010).

local continuum) are between $\sim 0.01-0.4 \mu \mathrm{m}$. These values are much lower than those typical of high metallicity star-forming galaxies (see Hernán-Caballero \& Hatziminaoglou 2011). This is expected given that we are probing smaller regions around the nucleus, and it is probable that the continuum emission is mostly arising from dust heated by the AGN.

To study a possible extranuclear origin for the $\mathrm{PAH}$ feature we investigated the morphology of the galaxies in our sample. We compiled the $b / a$ axial ratio (measurements from NED, RC3 $D_{25} / R_{25}$ isophotal $B$-band diameters) to determine the inclination of the host galaxy, where $b$ and $a$ are the minor and major axis, respectively. Axial ratios $b / a<0.5$ are considered as edge-on galaxies, whereas face-on galaxies have $b / a>0.5$ (see Table 1). With this definition we find 11 edge-on and 18 face-on galaxies. Out of the 13 sources with detection of the nuclear $11.3 \mu \mathrm{m}$ PAH feature, we find 5 (44\%) edge-on and 8 $(45 \%)$ face-on galaxies. We do not find that a positive detection predominates in edge-on galaxies, where material of the host galaxy along our line of sight could be misinterpreted as nuclear SF. However, we cannot rule out a dominant contribution from extranuclear SF in the most edge-on galaxies in our sample. González-Martín et al. (2013) found that the host galaxies could significantly contribute to the nuclear component for sources with the deepest silicate absorption features.

The majority of the nuclear $11.3 \mu \mathrm{m}$ PAH detections in our sample are galaxies with well-documented nuclear starbursts and/or recent SF activity based on UV and optical observations (González Delgado et al. 1998, NGC 5135, NGC 7130), modeling of the optical spectra (Storchi-Bergmann et al. 2000, NGC 5135, NGC 5643, NGC 7130, NGC 7582), and near-IR integral field spectroscopy (Davies et al. 2007; Tacconi-Garman \& Sturm 2013, Circinus, NGC 1068, NGC 3227, NGC 3783, NGC 5128).

\subsection{Stacking Nuclear Spectra with Undetected 11.3 $\mu \mathrm{m}$ PAH Emission}

In this section, we further investigate those galaxies with weak or no detected $11.3 \mu \mathrm{m}$ PAH feature emission. We stacked the individual spectra deemed to have undetected $11.3 \mu \mathrm{m}$ PAH features according to our $2 \sigma$ criterion (see Table 3 and Section 3.1). We divided them in two groups. The first includes galaxies with a weak silicate feature: ESO 323-G077, NGC 1365, NGC 3081, NGC 3783, NGC 4151, and NGC 4507. The second group contains galaxies with relatively deep silicate features: NGC 1386, NGC 2992, NGC 3281, NGC 4388, IC 5063, NGC 7172, and NGC 7479. We excluded from the stacking NGC 2110 and NGC 7213 because the silicate feature is strongly in emission and excluded NGC 5347 because the spectrum is very noisy. We normalized the spectra at $12 \mu \mathrm{m}$ and then used the IRAF task scombine with the average option to combine the different observations. 


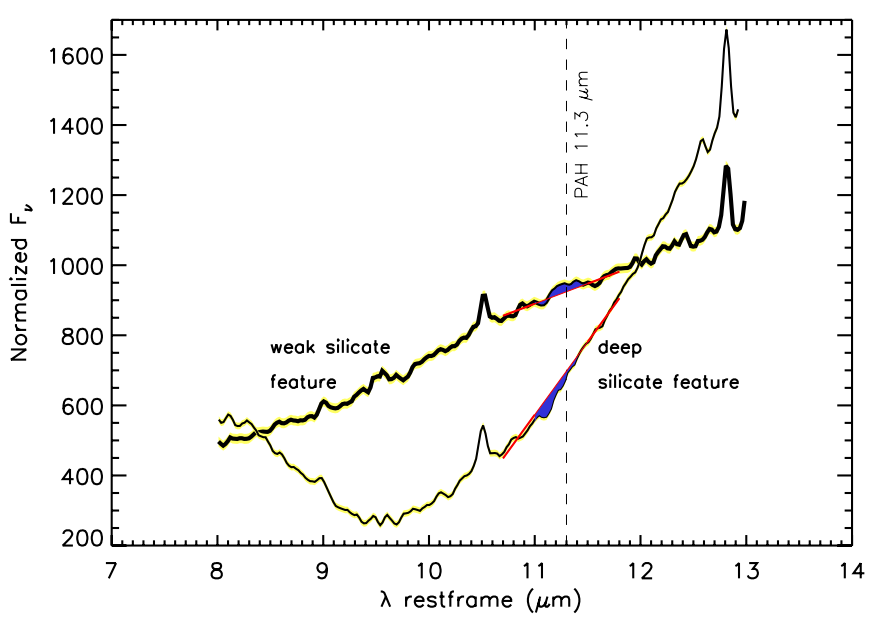

Figure 3. Result from the stacking of nuclear spectra (from T-ReCS, Michelle, and VISIR) without $11.3 \mu \mathrm{m}$ PAH detections. The stacked spectrum for sources with weak silicate features (six galaxies, thick line) shows a $2 \sigma$ detection of the $11.3 \mu \mathrm{m}$ PAH feature. In the stacked spectrum (thin line) of the seven galaxies with deep silicate features the PAH feature remains undetected. The flux density units are arbitrary. The individual spectra were normalized at $12 \mu \mathrm{m}$. See Section 3.2 for details.

(A color version of this figure is available in the online journal.)

Figure 3 shows the stacked spectra for the two groups. We applied the same method as in Section 3.1 to determine if the PAH feature is detected. We found that the $11.3 \mu \mathrm{m}$ PAH appears detected in the stacked nuclear spectrum of the galaxies with weak silicate features at a $2 \sigma$ level. The derived EW of the $11.3 \mu \mathrm{m}$ PAH is $8 \times 10^{-3} \mu \mathrm{m}$. The feature remains undetected in the stacked nuclear spectrum of sources with deep silicate features. This could be explained in part as due to extinction effects, given that the silicate absorption in these galaxies likely comes from cold foreground material (Goulding et al. 2012). We also note that the minimum around $11 \mu \mathrm{m}$ in the stacked spectrum of galaxies with deep silicate features could be from crystalline silicates. Indeed, Colling (2011) found that inclusion of crystalline silicates improved the fit of silicate feature in NGC 7172, NGC 7479, and NGC 4388. Therefore, it would be expected to appear in the stacked spectrum.

\subsection{Is the 11.3 $\mu \mathrm{m}$ PAH Feature Suppressed in the Vicinity of AGN?}

It has been known for more than $20 \mathrm{yr}$ now that PAH emission is weaker in local AGN than in high metallicity star-forming galaxies, although some AGN do also show strong PAH features on circumnuclear scales (Roche et al. 1991). It is not clear, however, if the decreased detection of PAH emission and the smaller EWs of the PAH features in AGN are due to (1) an increased mid-IR continuum arising from the AGN, (2) the destruction of the PAH carriers in the harsh environment near the AGN (Roche et al. 1991; Voit 1992a), or (3) decreased SF in the nuclear region (Hönig et al. 2010). Additionally, there is a prediction that smaller PAH molecules would be destroyed more easily in strong radiation fields (see, e.g., Siebenmorgen et al. 2004), also indicating that different PAHs may behave differently.

The effects of an increasing continuum produced by the AGN is clearly seen in the $3.3 \mu \mathrm{m}$ PAH map of the central region of NGC 5128. The ratio of the feature-to-continuum (i.e., the $\mathrm{EW}$ of the feature) decreases toward the AGN, whereas the feature peaks in the center (see Tacconi-Garman \& Sturm 2013 for more details). This implies that the PAH molecules

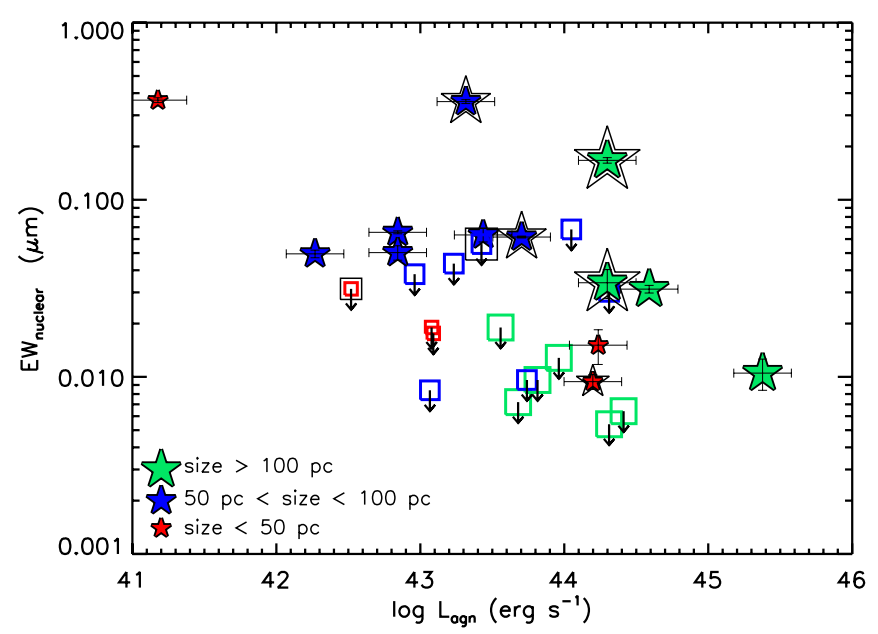

Figure 4. Nuclear EW of the $11.3 \mu \mathrm{m}$ PAH feature vs. the AGN bolometric luminosity. Filled star symbols are detections, whereas open squares are upper limits. The sizes/colors of the symbols (see figure legend) indicate the different physical sizes probed, which are determined by the slit widths of the observations. Hereinafter, we have marked Compton-thick objects in all plots using a double-star or a double-square for detections or upper limits, respectively.

(A color version of this figure is available in the online journal.)

are not destroyed in the harsh environment around the AGN of this galaxy (see also Section 3.4). Similarly, Díaz-Santos et al. (2010) showed that at least the molecules responsible for the $11.3 \mu \mathrm{m}$ PAH feature can survive within $<100 \mathrm{pc}$ from the AGN.

Some recent observational works reached apparently opposing conclusions on the PAH emission of AGN on physical scales within a few kpc from the nucleus. But note that this is for much larger physical scales than those probed here. Diamond-Stanic \& Rieke (2010) demonstrated for the RSA Seyferts that the $11.3 \mu \mathrm{m}$ PAH feature is not suppressed, whereas other mid-IR PAH features are. LaMassa et al. (2012), on the other hand, combined optical and mid-IR spectroscopy of a large sample of AGN and star-forming galaxies and concluded that in AGN-dominated systems (higher luminosity AGN) the $11.3 \mu \mathrm{m}$ PAH feature does get suppressed.

To investigate this issue, in Figure 4 we plotted the observed nuclear EW of the $11.3 \mu \mathrm{m}$ PAH feature against the AGN bolometric luminosity for our sample, with smaller symbols indicating regions closer to the AGN. This figure does not show any clear trend. If the decreased nuclear EW of the 11.3 $\mu \mathrm{m}$ PAH feature were due to the AGN mid-IR continuum in more luminous AGN, we would expect a trend of decreasing EW for increasing AGN bolometric luminosities. Alternatively, we would expect the same trend if the PAH molecules responsible for the $11.3 \mu \mathrm{m}$ feature were suppressed/destroyed more easily in luminous AGN. From this figure we can see that at a given AGN luminosity we sometimes detect nuclear $11.3 \mu \mathrm{m}$ PAH emission, whereas in other cases we do not. In other words, we do not see clear evidence in our sample for the $11.3 \mu \mathrm{m}$ PAH feature to be suppressed in more luminous AGN, at least for the AGN bolometric luminosities covered by our sample of Seyfert galaxies.

As can be seen from Figure 4, there is no clear influence of the physical region size probed on the observed EW. Hence, we do not see a tendency for the EW of the PAH feature to decrease for smaller physical regions. This would be the case if we were to expect a higher AGN continuum contribution and/or PAH destruction as we get closer to the AGN. No trend is present 
when plotting the observed EWs with respect to luminosity densities. We note, however, that for the three closest Seyferts (Circinus, NGC 4945, NGC 5128) the $11.3 \mu \mathrm{m}$ PAH feature is not detected in ground-based high-resolution T-ReCS spectra, which probe scales of $\sim 7-15$ pc for these sources (Roche et al. 2006; González-Martín et al. 2013).

\subsection{Nuclear PAH Molecules Shielded by the Dusty Torus?}

As we have shown in the previous sections and as presented by others (e.g., Miles et al. 1994; Marco \& Brooks 2003; DíazSantos et al. 2010; Hönig et al. 2010; González-Martín et al. 2013; Sales et al. 2013; Tacconi-Garman \& Sturm 2013) PAH emission is detected in the vicinity (ten to a few hundred parsecs) of the harsh environments of some AGN. Therefore, at least in some galaxies, the PAH molecules are not destroyed (or at least not completely) near the AGN. Therefore, they must be shielded from the AGN by molecular material with sufficient X-ray absorbing column densities (Voit 1992a; Miles et al. 1994; Watabe et al. 2008). As pointed out by Voit (1992b), for PAH features to be absent due to destruction, they have to be fragmented more quickly than they can be rebuilt. In other words, PAHs will exist if the rate of reaccretion of carbon onto PAHs is higher than the evaporation rate caused by the AGN. Using the parameters of the Voit (1991) model, Miles et al. (1994) estimated the column density of hydrogen required to keep the evaporation rate of PAHs below the rate of reaccretion of carbon onto the PAHs. As derived in Miles et al. (1994), the timescale for X-ray absorption in terms of the hydrogen column density of the intervening material $N_{\mathrm{H}}($ tot), the distance from the AGN fixed in our case by the slit width $D_{\text {agn }}$, and the X-ray luminosity of the AGN, can be written as

$$
\tau \approx 700 \mathrm{yr}\left(\frac{N_{\mathrm{H}}(\mathrm{tot})}{10^{22} \mathrm{~cm}^{-2}}\right)^{1.5}\left(\frac{D_{\mathrm{agn}}}{\mathrm{kpc}}\right)^{2}\left(\frac{10^{44} \mathrm{erg} \mathrm{s}^{-1}}{L_{\mathrm{X}}}\right) .
$$

Voit (1992b) estimated that the time scale needed for reaccretion of a carbon atom on to a fractured PAH should be at least $3000 \mathrm{yr}$ for the typical conditions of the interstellar medium (ISM).

The protecting material, which has to be located between the nuclear sites of SF and the AGN, is likely to be that in the dusty torus postulated by the unified model. In a number of works (Ramos Almeida et al. 2009, 2011; Alonso-Herrero et al. 2011, 2012) we have demonstrated that the clumpy torus models of Nenkova et al. (2008a, 2008b) accurately reproduce the nuclear infrared emission of local Seyfert galaxies. These models are defined by six parameters describing the torus geometry and the properties of the dusty clouds. These are the viewing angle $(i)$ and radial extent $(Y)$ of the torus, the angular $(\sigma)$ and radial distributions $(q)$ of the clouds along with its optical depth $\left(\tau_{\mathrm{V}}\right)$, and the number of clouds along the equatorial direction $\left(N_{0}\right)$. The optical extinction of the torus along the line of sight is computed from the model parameters as $A_{V}^{\mathrm{LOS}}=1.086 N_{0} \tau_{\mathrm{V}} e^{\left(-(i-90)^{2} / \sigma^{2}\right)} \mathrm{mag}$. According to Bohlin et al. (1978), the absorbing hydrogen column density is then calculated following $N_{\mathrm{H}}^{\mathrm{LOS}} / A_{V}^{\mathrm{LOS}}=1.9 \times 10^{21} \mathrm{~cm}^{-2} \mathrm{mag}^{-1}$.

With the derived torus model parameters, we estimated the hydrogen column density of the torus material in our line of sight for a sample of Seyferts. This typically ranges from $N_{\mathrm{H}}^{\mathrm{LOS}} \simeq$ $10^{23}$ to a few times $10^{24} \mathrm{~cm}^{-2}$ (see Ramos Almeida et al. 2009, 2011; Alonso-Herrero et al. 2011, 2012 for further details). Using Equation (1) for the hard X-ray luminosities (Table 1) and distances from the AGN probed by our spectroscopy (see Table 3), and setting $\tau=3000 \mathrm{yr}$ as proposed by Voit (1992a), we require hydrogen column densities of at least a few $10^{23} \mathrm{~cm}^{-2}$ to protect the PAHs from the AGN radiation. Evidence for such values for the $N_{\mathrm{H}}$ are found for our sample, as we derived absorbing columns of the order of $10^{23} \mathrm{~cm}^{-2}$ or even higher.

In Ramos Almeida et al. (2011), we also demonstrated that Seyfert 2s are more likely to have higher covering factors than Seyfert 1s. Assuming that the nuclear SF occurs inside the torus, the PAH molecules may be more shielded in the nuclear region of Seyfert 2s. However, even the $N_{\mathrm{H}}$ values along our line of sight from the torus model fits of Seyfert 1s (which would be a lower limit to the total $N_{\mathrm{H}}$ in the torus) with $11.3 \mu \mathrm{m}$ PAH detections are sufficient to protect the PAH carriers. This is the case for four Seyfert 1s (NGC 3227, NGC 5506, NGC 7469, and NGC 7582), as can be seen from the modeling by AlonsoHerrero et al. (2011). Note that the column densities that we refer to are not only those absorbing the X-rays but also those including material much farther away from the accreting $\mathrm{BH}$.

Another interesting aspect to keep in mind from Equation (1) is that the column densities needed to protect the PAH molecules from the AGN X-ray emission become higher for more luminous AGN as well as for distances closer to the AGN. However, we emphasize that for the AGN luminosities of the RSA Seyferts and distances from the AGN probed by the observations presented here, the PAH molecules are likely to be shielded from the AGN by material in the torus residing on smaller scales. Also, part of the obscuring material even on these nuclear scales can reside in the host galaxy as shown by González-Martín et al. (2013). Thus, another source of opacity that might prevent the PAHs from being destroyed are dust lanes in galaxies or dust in the nuclear regions of merger systems. This might be the case for five sources in our sample, namely NGC 4945, NGC 5128, NGC 5506, NGC 7130, and NGC 7582.

\section{NUCLEAR STAR FORMATION RATES IN SEYFERT GALAXIES}

\subsection{Relation between the 11.3 $\mathrm{m}$ PAH Feature and the [Ne II] $12.81 \mu \mathrm{m}$ Emission Line on Nuclear Scales}

In star-forming galaxies the luminosity of the [Ne II] $12.81 \mu \mathrm{m}$ emission line is a good indicator of the SFR (Roche et al. 1991; Ho \& Keto 2007; Díaz-Santos et al. 2010). We note that these measurements are contaminated by the $12.7 \mu \mathrm{m}$ PAH feature, which is not easily resolvable.

In Seyfert galaxies the situation is more complicated because this line can be excited by both SF and AGN activity. The AGN contribution to the [Ne II] varies from galaxy to galaxy in local Seyfert galaxies and other AGN (see, e.g., Meléndez et al. 2008; Pereira-Santaella et al. 2010). For the RSA Seyfert sample, Diamond-Stanic \& Rieke (2010) used IRS spectroscopy to compare the circumnuclear SFRs computed with the [Ne II] line and the $11.3 \mu \mathrm{m}$ PAH feature as a function of $\mathrm{EW}$ of the PAH. They found that the ratio of the two circumnuclear SFRs (on kpc scale) is on average unity, with some scatter for galaxies with large PAH EWs $>0.3 \mu \mathrm{m}$. The most discrepant measurements were for galaxies with elevated [O IV] $/[\mathrm{Ne}$ II $]$ and low EW of the PAH, that is, AGN-dominated galaxies.

In Figure 5 (left panel), we show the observed nuclear $\mathrm{PAH} /[\mathrm{Ne}$ II] ratio as a function of the AGN luminosity. To correct for the $12.7 \mu \mathrm{m}$ PAH contamination, we have used a median ratio of the 11.3 versus the $12.7 \mu \mathrm{m}$ PAH features of 1.8, derived in Smith et al. (2007) and subtracted it from the [Ne II] measurement. NGC 1365, NGC 1386, and NGC 5347 are not included in the plot because both lines are undetected and, 

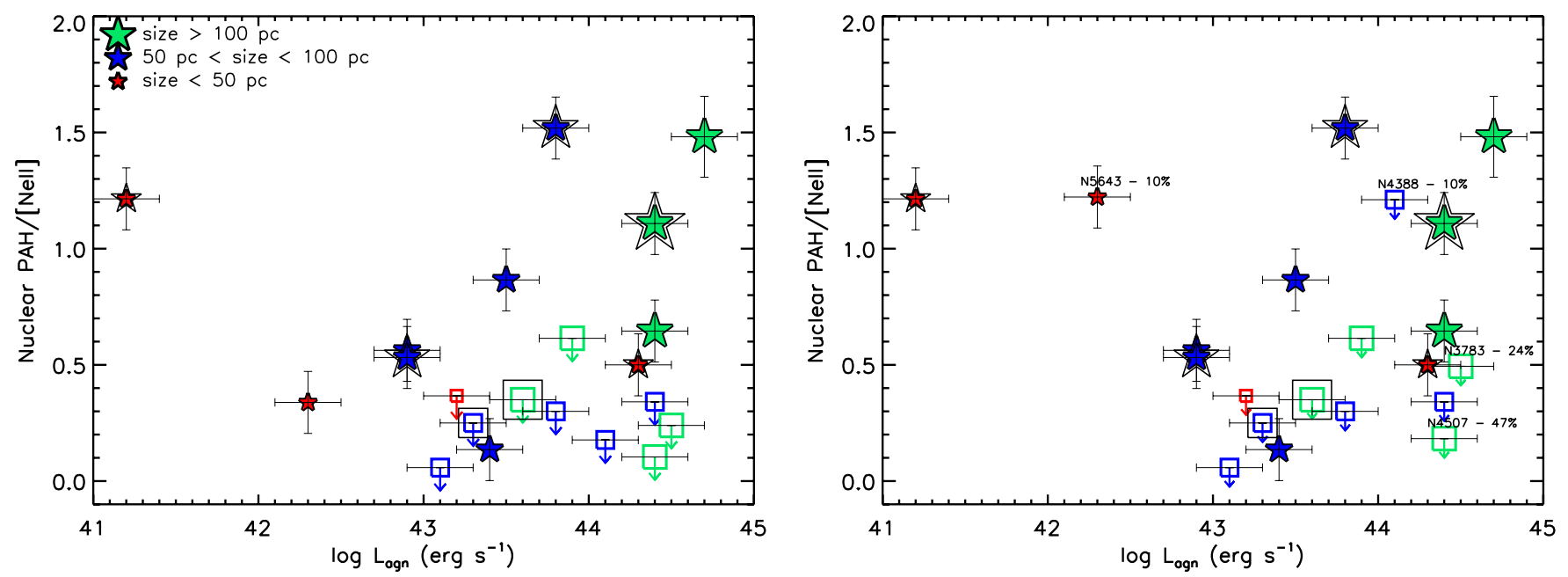

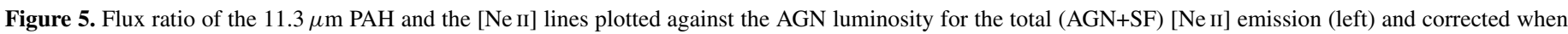

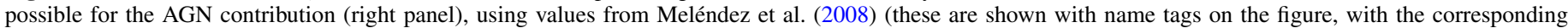
percentage of star formation). See the text for details.

(A color version of this figure is available in the online journal.)

therefore, the value in the $Y$ axis is completely unconstrained. We derived the [Ne II] fluxes using the same technique as explained for the PAH in Section 2.3, integrating the line between 12.6 and $12.9 \mu \mathrm{m}$. For seven galaxies, namely IC 5063, Mrk 509, NGC 1068, NGC 2110, NGC 5135, NGC 7130, and NGC 7479, the [Ne II] line falls outside the wavelength range covered by our observations. They are not included in the plot except for NGC 5135 and NGC 7130, whose values have been extracted from Díaz-Santos et al. (2010). As can be seen from this figure, most of the galaxies with detections of the nuclear $11.3 \mu \mathrm{m}$ PAH feature and [Ne II] show ratios similar to those of high metallicity star-forming galaxies $(\sim 0.7-2$, see, e.g., Roche et al. 1991; Díaz-Santos et al. 2010), even if the [Ne II] fluxes are not corrected for AGN emission. On the other hand, most of the nuclear spectra with nondetections show upper limits to the $\mathrm{PAH} /[\mathrm{Ne}$ II $]$ ratio below 0.5 .

To derive the nuclear [Ne II] flux solely due to SF, we can use the fractional SF contribution to the [Ne II] line within the IRS aperture estimated by Meléndez et al. (2008). For sources with strong AGN contribution (higher than 50\%), we estimated the [Ne II] flux coming from the AGN, which in turn can be subtracted from the observed nuclear [Ne II] flux. This is shown in Figure 5 (right panel). We note that for NGC 2992, NGC 3227, NGC 4151, NGC 5506, and NGC 7172, the estimated AGN contribution to the total [Ne II] is higher than the nuclear [Ne II] value, indicating that the AGN [Ne II] contributions were overestimated. For these galaxies, we did not apply any correction. Figure 5 shows that for those Seyferts with a nuclear $11.3 \mu \mathrm{m}$ PAH detection the $\mathrm{PAH} /[\mathrm{Ne} \mathrm{II}]_{\mathrm{SF}}$ ratio does not decrease with the AGN bolometric luminosity. This would be expected if the PAH emission was to be suppressed. Therefore, given that the $\mathrm{PAH} /[\mathrm{Ne} \mathrm{II}]_{\mathrm{SF}}$ ratio does not show a dependence on $L_{\text {agn }}$, we conclude that the $11.3 \mu \mathrm{m}$ PAH feature emission can be used to estimate the nuclear SFRs (see next section).

\subsection{Circumnuclear $(\sim 600 p c)$ versus Nuclear $(\sim 65 p c)$ Scales}

In the vicinity of an AGN, we expect the chemistry and/or the heating as dominated by X-rays from the so-called
X-ray dominated regions (XDRs). In principle, XDRs could also contribute to $\mathrm{PAH}$ heating through the photodissociation and photoionization by FUV photons produced via excitation of $\mathrm{H}$ and $\mathrm{H}_{2}$ in collisions with secondary electrons. However, as we derived in Section 3.4, the torus appears to provide the appropriate environment to shield the PAH molecules from the AGN emission on typical physical scales of a few parsecs up to a few tens of parsecs. We thus expect little or no contribution of UV AGN produced photons to the $\mathrm{PAH}$ heating in the nuclear scales of Seyfert galaxies. Hereinafter, we assume that the aromatic molecules are heated by the radiation field produced by young massive stars and that the $11.3 \mu \mathrm{m}$ PAH luminosity can be used to estimate the SFR.

We derived nuclear and circumnuclear SFRs using the PAH $11.3 \mu \mathrm{m}$ feature luminosities and applying the relation derived in Diamond-Stanic \& Rieke (2012)

$$
\operatorname{SFR}\left(M_{\odot} \mathrm{yr}^{-1}\right)=9.6 \times 10^{-9} L\left(\mathrm{PAH}_{11.3 \mu \mathrm{m}}, L_{\odot}\right)
$$

using PAHFIT measurements of galaxies with IR $(8-1000 \mu \mathrm{m})$ luminosities $L_{\mathrm{IR}}<10^{11} L_{\odot}$, using the Rieke et al. (2009) templates and a Kroupa initial mass function. This is then appropriate for our sample, as the median value of the IR luminosity of the individual galaxies is $5 \times 10^{10} L_{\odot} \cdot{ }^{17}$ The uncertainties in the derived SFRs using Equation (2) are typically 0.28 dex (see Diamond-Stanic \& Rieke 2012 for full details).

For the 13 galaxies with nuclear $11.3 \mu \mathrm{m}$ PAH detections, the nuclear SFRs span two orders of magnitude between $\sim 0.01-1.2 M_{\odot} \mathrm{yr}^{-1}$ for regions of typically $\sim 65 \mathrm{pc}$ in size. The nondetections indicate that the nuclear SFR of RSA Seyfert galaxies from the high angular resolution spectroscopy can be $\sim 0.01-0.2 M_{\odot} \mathrm{yr}^{-1}$, or even lower.

The projected nuclear SFR densities are between 2 and $93 M_{\odot} \mathrm{yr}^{-1} \mathrm{kpc}^{-2}$ with a median value $22 M_{\odot} \mathrm{yr}^{-1} \mathrm{kpc}^{-2}$. These are consistent with the simulations of Hopkins et al. (2012) for similar physical scales. The two galaxies with the largest SFR densities are NGC 1068 and NGC 1808 with values of

\footnotetext{
17 A few galaxies in our sample have total IR luminosities between $10^{11}-5 \times 10^{11} L_{\odot}$. However, given that we are dealing with nuclear luminosities, we expect those being less than the $10^{11} L_{\odot}$ limiting value.
} 


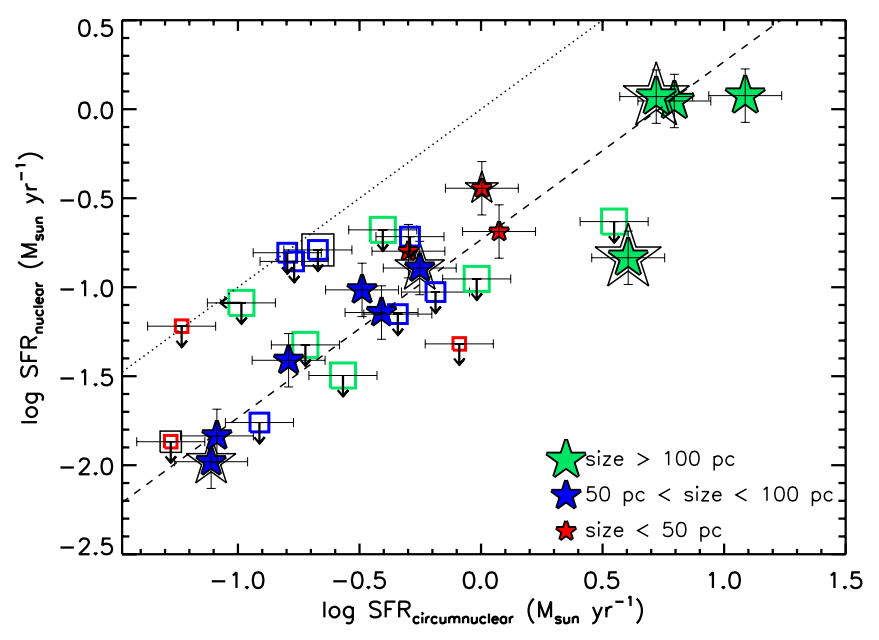

Figure 6. Comparison between the SFR on different scales, where $\mathrm{SFR}_{\text {circumnuclear }}$ implies typical physical scales of $\sim 0.6 \mathrm{kpc}$ and $\mathrm{SFR}_{\text {nuclear }}$ is for $\sim 65$ pc scales. Nondetections are plotted at a $2 \sigma$ level. Symbols as in Figure 4. The dashed line shows the median value for the nuclear/circumnuclear SFR ratio for the detections of the nuclear PAH feature (see text and Table 3). The dotted line indicates a nuclear/circumnuclear ratio of one.

(A color version of this figure is available in the online journal.)

414 and $329 M_{\odot} \mathrm{yr}^{-1} \mathrm{kpc}^{-2}$, respectively. We notice that for those galaxies in common with Davies et al. (2007; Circinus, NGC 1068, NGC 3783, NGC 7469, and NGC 3227), we find quite discrepant values for the SFR density, with our values lying below those in Davies et al. (2007) except for NGC 1068. It might be due to the use of different SF histories and SFR indicators. We note that with the $11.3 \mu \mathrm{m}$ PAH feature we cannot explore age effects (see Díaz-Santos et al. 2008; Figure 8) as this feature can be excited by both $\mathrm{O}$ and $\mathrm{B}$ stars, and thus it integrates over ages of up to a few tens of millions of years (Peeters et al. 2004), unlike the measurements in Davies et al. (2007) that sample younger populations. In addition, we detect neither nuclear nor circumnuclear SF in NGC 3783 based on the PAH measurements. On the other hand, PAHs can also be found in the ISM as being excited in less-UV rich environments, such as reflection nebulae (e.g., Li \& Draine 2002). However, the decreased strength of the IR emission features in these objects seems to indicate the low efficiency of softer near-UV or optical photons in exciting PAHs in comparison to SF (Tielens 2008).

The circumnuclear SFRs of the Seyferts in our sample are between 0.2 and $18.4 M_{\odot} \mathrm{yr}^{-1}$ (see also Diamond-Stanic \& Rieke 2012), and the median circumnuclear SFR densities are $1.2 M_{\odot} \mathrm{yr}^{-1} \mathrm{kpc}^{-2}$. These are similar to those of Seyfert galaxies in the CfA (Huchra \& Burg 1992) and $12 \mu \mathrm{m}$ (Rush et al. 1993) samples, as derived using the $3.3 \mu \mathrm{m}$ PAH feature (see Imanishi 2003; Imanishi \& Wada 2004).

The comparison between the nuclear and circumnuclear SFRs for our sample clearly shows that, in absolute terms, the nuclear SFRs are much lower (see Table 3). This is in good agreement with previous works based on smaller samples of local AGN (e.g., Siebenmorgen et al. 2004; Watabe et al. 2008; Hönig et al. 2010; González-Martín et al. 2013). The median value of the ratio between the nuclear and circumnuclear SFRs for the detections of the $11.3 \mu \mathrm{m}$ feature is $\sim 0.18$ (see also Table 3 ), with no significant difference for type 1 and type 2 Seyferts ( $\sim 0.18$ and $\sim 0.21$, respectively).

In Figure 6, we plot the nuclear and circumnuclear SFRs probing typical physical scales of $\sim 65 \mathrm{pc}$ and $\sim 600 \mathrm{pc}$, respectively. Again, nondetections are plotted as upper limits

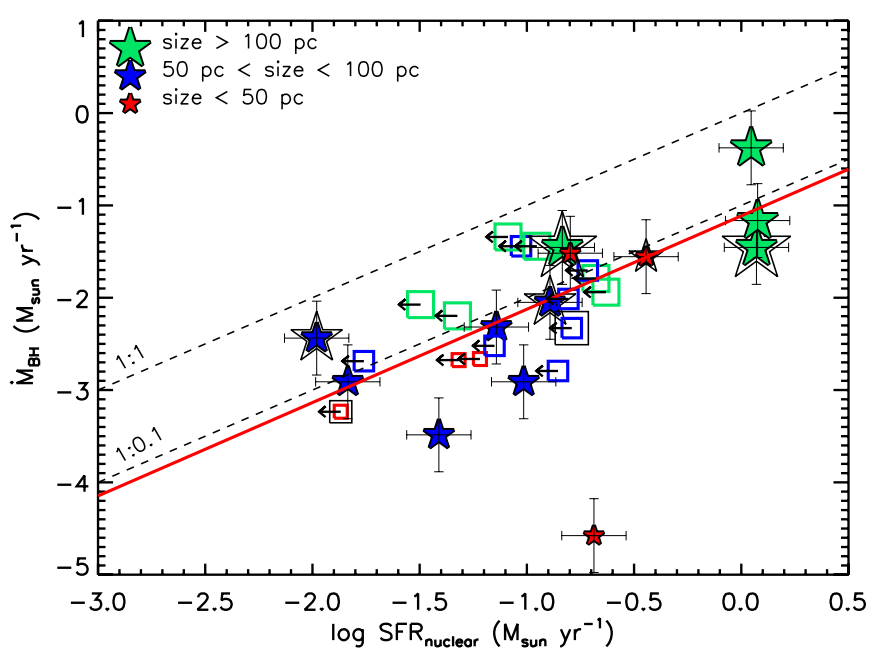

Figure 7. Observed nuclear SFR vs. $\dot{M}_{\mathrm{BH}}$ relation. Predictions from Hopkins \& Quataert (2010) are shown as dashed lines. We show the $\dot{M}_{\mathrm{BH}} \approx 0.1 \times \mathrm{SFR}$ relation, which is expected for $r<100 \mathrm{pc}$, and the $1: 1$ relation which, is expected for the smallest physical scales $(r<10 \mathrm{pc})$. The solid line represents the fit to our detections of the nuclear $11.3 \mu \mathrm{m}$ PAH feature (see text for details).

(A color version of this figure is available in the online journal.)

at the $2 \sigma$ level. Overall, for our detections, the fraction of the SFR accounted for by the central $\sim 65$ pc region of our Seyferts ranges between $\sim 5 \%-35 \%$ of that enclosed within the aperture corresponding to the circumnuclear data.

While the nuclear SFRs are lower than the circumnuclear SFRs, the median nuclear projected SFR densities are approximately a factor of 20 higher than the circumnuclear ones in our sample (median values of 22 and $1 M_{\odot} \mathrm{yr}^{-1} \mathrm{kpc}^{-2}$, respectively). This shows that the SF is not uniformly distributed, but it is concentrated in the nuclear regions of the RSA Seyferts studied here. This is in agreement with simulations of Hopkins et al. (2012). The molecular gas needed to maintain these nuclear SFR densities appears to have higher densities in Seyfert galaxies than those of quiescent (non-Seyferts) galaxies (Hicks et al. 2013).

\subsection{Nuclear Star Formation Rate Versus Black Hole Accretion Rate}

Hopkins \& Quataert (2010) performed smoothed particle hydrodynamic simulations to study the inflow of gas from galactic scales $(\sim 10 \mathrm{kpc})$ down to $\lesssim 0.1 \mathrm{pc}$, where key ingredients are gas, stars, BHs, self-gravity, SF, and stellar feedback. These numerical simulations indicate a relation (with significant scatter) between the SFR and $\dot{M}_{\mathrm{BH}}$ that holds for all scales, and it is more tightly coupled for the smaller physical scales. The model of Kawakatu \& Wada (2008) predicts that the AGN luminosity should also be tightly correlated with the luminosity of the nuclear (100 pc) SF in Seyferts and QSOs, and also that $L_{\text {nuclear,SB }}$ $L_{\mathrm{AGN}}$ is larger for more luminous AGN.

According to Alexander \& Hickox (2012, and references therein), $\dot{M}_{\mathrm{BH}}$ and AGN luminosities follow the relation

$$
\dot{M}_{\mathrm{BH}}\left(M_{\odot} \mathrm{yr}^{-1}\right)=0.15(0.1 / \epsilon)\left(L_{\mathrm{agn}} / 10^{45} \mathrm{erg} \mathrm{s}^{-1}\right),
$$

where we used $\epsilon=0.1$ as the typical value for the mass-energy conversion efficiency in the local universe (Marconi et al. 2004). We obtained $\dot{M}_{\mathrm{BH}}$ ranging between $5 \times 10^{-6}$ and $0.5 M_{\odot} \mathrm{yr}^{-1}$ for our sample of Seyfert galaxies. Uncertainties in the $\dot{M}_{\mathrm{BH}}$ estimations are dominated by those in $L_{\mathrm{agn}}$, i.e., $0.4 \mathrm{dex}$ as mentioned in Section 2.1. 
Figure 7 shows the observed nuclear SFR against $\dot{M}_{\mathrm{BH}}$ for the Seyferts in our sample. The different sizes of the symbols indicate different physical sizes of the probed regions. The prominent outlier in this figure is NGC $1808 .{ }^{18} \mathrm{We}$ also show in Figure 7 as dashed lines predictions from the Hopkins \& Quataert (2010) simulations for $r<100$ pc $\dot{M}_{\mathrm{BH}} \approx 0.1 \times \operatorname{SFR}(r<100 \mathrm{pc})$ and $r<10 \mathrm{pc}\left(\dot{M}_{\mathrm{BH}} \approx \mathrm{SFR}\right)$. These radii encompass approximately the physical scales probed by our nuclear SFR. The prediction from the Kawakatu \& Wada (2008) disk model for Seyfert luminosities and BH masses similar to those of our sample falls between the two dashed lines $(\sim 1: 0.4$ relation).

To derive a possible correlation between the nuclear SFR and $\dot{M}_{\mathrm{BH}}$, we applied a simple fit relation (slope of 1.01 and uncertainties of 0.4 dex in both parameters, Equation (4)), which is close to the 1:0.1 relation (see Figure 7 and below).

$$
\log \dot{M}_{\mathrm{BH}}=1.01 \times \log \mathrm{SFR}_{\text {nuclear }}-1.11
$$

Also including the upper limits in the fit we obtained a very similar result (slope of 0.95). In contrast, Diamond-Stanic \& Rieke (2012) obtained a slightly superlinear relation $\left(\dot{M}_{\mathrm{BH}} \propto\right.$ $\left.\operatorname{SFR}(r<1 \mathrm{kpc})^{1.6}\right)$, when the SFR are measured in regions of $1 \mathrm{kpc}$ radius. This behavior (i.e., the relations becoming linear on smaller scales) is nevertheless predicted by the Hopkins \& Quataert (2010) simulations.

As can be seen from Figure 7, the Hopkins \& Quataert (2010) predictions for $r<100 \mathrm{pc}$ reproduce the observed relation for our sample fairly well. We do not find a tendency for galaxies with SFR measured in regions closer to the AGN (slit sizes of less than $100 \mathrm{pc}$ ) to have larger $\dot{M}_{\mathrm{BH}}$ to SFR ratios (i.e., to lie closer to the 1:1 relation) than the rest, as predicted by Hopkins \& Quataert (2010). It is worth noting that these authors caution that their work do not include the appropriate physics for low accretion rates $\left(\ll 0.1 M_{\odot} \mathrm{yr}^{-1}\right)$. The scatter in the theoretical estimations and the limited size of our sample of Seyfert galaxies prevent us from further exploring this issue. Future planned observations with the mid-IR CanariCam (Telesco et al. 2003) instrument on the $10.4 \mathrm{~m}$ Gran Telescopio de Canarias will allow a similar study for larger samples of Seyfert galaxies.

\section{SUMMARY AND CONCLUSIONS}

We have presented the largest compilation to date of high angular resolution $\left(0^{\prime \prime} 4-0.0^{\prime} 8\right)$ mid-IR spectroscopy of nearby Seyfert galaxies obtained with the T-ReCS, VISIR, and Michelle instruments. We used the $11.3 \mu \mathrm{m}$ PAH feature to study the nuclear SF activity and its relation to the circumnuclear SF, as well as with $\dot{M}_{\mathrm{BH}}$. The sample includes 29 Seyfert galaxies (13 Seyfert 1 and 16 Seyfert 2 galaxies) belonging to the nearby RSA AGN sample (Maiolino \& Rieke 1995; Ho et al. 1997). It covers more than two orders of magnitude in AGN bolometric luminosity, with the galaxies located at a median distance of $33 \mathrm{Mpc}$. Our data allow us to probe typical nuclear physical scales (given by the slit widths) of $\sim 65 \mathrm{pc}$. We used the hard X-ray luminosity as a proxy for the AGN bolometric luminosity and $\dot{M}_{\mathrm{BH}}$. We used mid-infrared Spitzer/IRS spectroscopy to

\footnotetext{
18 This galaxy is a low-luminosity AGN and shows the lowest AGN bolometric luminosity in our sample. It seems plausible that the SF, unlike the AGN as for the rest of the sample, is the dominant mechanism contributing to the nuclear mid-IR emission (see Mason et al. 2012), as this source is completely off the mid-IR versus hard X-ray correlation for AGN (see González-Martín et al. 2013).
}

study the SF taking place in the circumnuclear regions (a factor of 5-10 larger scales).

The main results can be summarized as follows.

1. The detection rate of the nuclear $11.3 \mu \mathrm{m}$ PAH feature in our sample of Seyferts is $45 \%$ (13 out of 29 sources), at a significance of $2 \sigma$ or higher. Additionally, the stacked spectra of six galaxies without a detection of the $11.3 \mu \mathrm{m}$ PAH feature and weak silicate features resulted into a positive detection of the $11.3 \mu \mathrm{m}$ PAH feature above $2 \sigma$.

2. There is no evidence of strong suppression of the nuclear $11.3 \mu \mathrm{m}$ PAH feature in the vicinity of the AGN, at least for the Seyfert-like AGN luminosities and physical nuclear regions (65 pc median value) sampled here. In particular, we do not see a tendency for the EW of the PAH to decrease for more luminous AGN. The hydrogen column densities predicted from clumpy torus model-fitting (a few $10^{23} \mathrm{~cm}^{-2}$ up to a few $10^{24} \mathrm{~cm}^{-2}$ ) would be, in principle, sufficient to shield the PAH molecules from AGN X-ray photons in our Seyfert galaxies.

3. The nuclear SFRs in our sample derived from the $11.3 \mu \mathrm{m}$ PAH feature luminosities are between 0.01 and 1.2 $M_{\odot} \mathrm{yr}^{-1}$, where we assumed no XDR-contribution to the PAH heating. There is a significant reduction of the $11.3 \mu \mathrm{m}$ PAH flux from circumnuclear (median size of $600 \mathrm{pc}$ ) to nuclear regions (median size of $65 \mathrm{pc}$ ), with a typical ratio of $\sim 5$. Although this indicates that the SFRs are lower near the AGN in absolute terms, the projected SFR rate density in the nuclear regions (median value of $22 M_{\odot} \mathrm{yr}^{-1} \mathrm{kpc}^{-2}$ ) is approximately 20 higher than in the circumnuclear regions. This indicates that the SF activity is highly concentrated in the nuclear regions in our sample of Seyfert galaxies.

4. Predictions from numerical simulations for the appropriate physical regions are broadly consistent with the observed relation between the nuclear SFR and $\dot{M}_{\mathrm{BH}}$ in our sample (slope of $1.01 \pm 0.4$ ). Although limited by the relatively small number of sources in our sample, we do not find decreased nuclear SFR-to- $\dot{M}_{\mathrm{BH}}$ ratios for regions closer to the AGN, as predicted by the Hopkins \& Quataert (2010) simulations.

We thank E. Piconcelli for useful discussion and the anonymous referee for comments that helped us to improve the article.

P.E. and A.A.H. acknowledge support from the Spanish Plan Nacional de Astronomía y Astrofísica under grant AYA200905705-E. P. E. is partially funded by the Spanish MINECO under grant AYA2012-39362-C02-01. A.A.H. and A.H.C. acknowledge support from the Augusto G. Linares Program through the Universidad de Cantabria. C.R.A. acknowledges financial support from the Spanish Plan Nacional de Astronomía y Astrofísica under grant AYA2010-21887-C04.04 (Estallidos). O.G.M. and J.M.R.E. acknowledge support from the Spanish MICINN through the grant AYA2012-39168-C03-01. S.F.H. acknowledges support by Deutsche Forschungsgemeinschaft (DFG) in the framework of a research fellowship (Auslandsstipendium).

This research has made use of the NASA/IPAC Extragalactic Database (NED), which is operated by the Jet Propulsion Laboratory, California Institute of Technology, under contract with the National Aeronautics and Space Administration. Our work is based on observations obtained at the Gemini Observatory, which is operated by the Association of Universities for Research in Astronomy, Inc., under a cooperative 

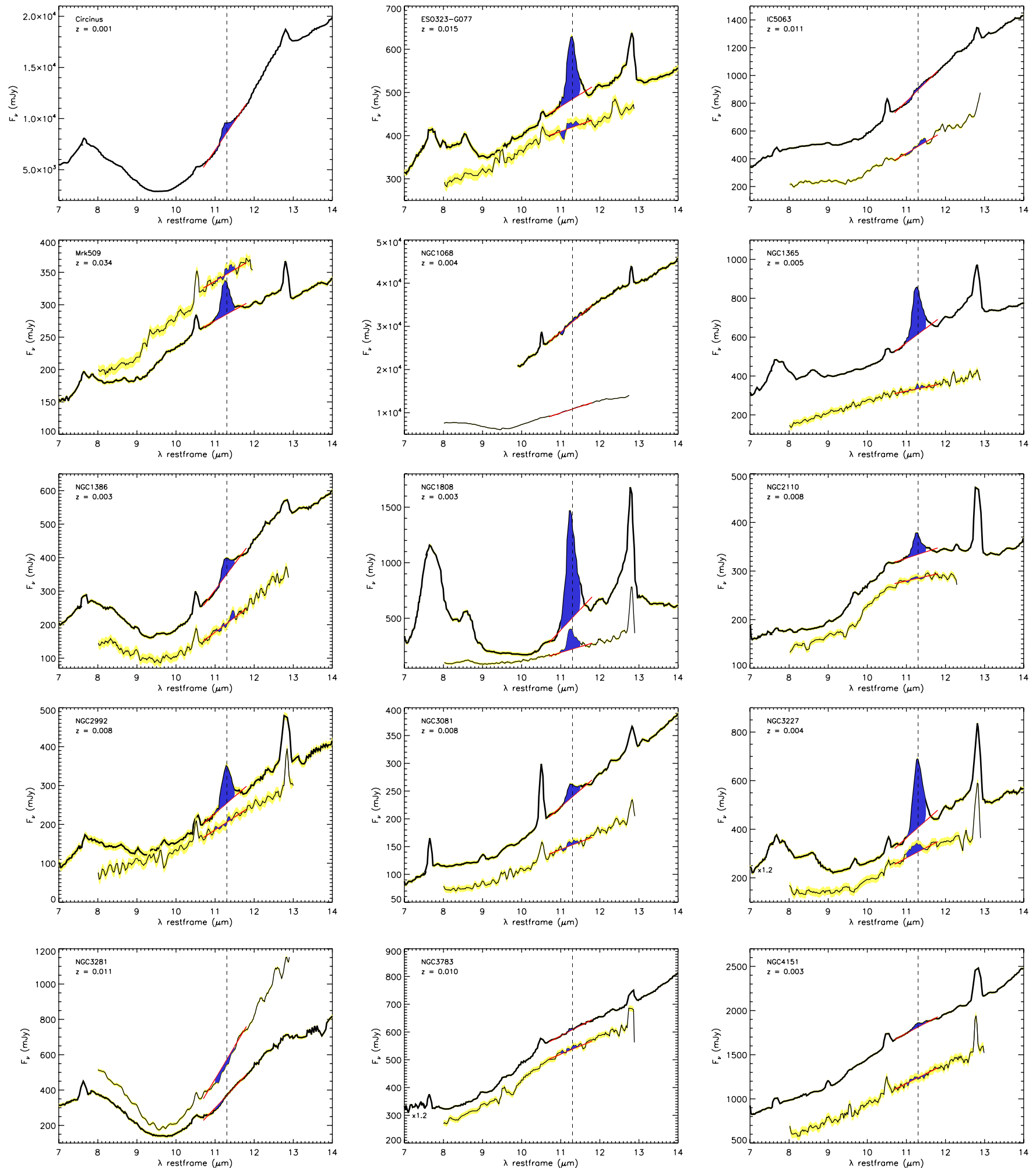

Figure 8. Spectra of the sample. Spitzer/IRS SL spectra (thick line) compared with the ground-based spectra (T-ReCS/VISIR/Michelle: thin line). All observations have been smoothed to the same spectral resolution. We show the location of the $11.3 \mu \mathrm{m} \mathrm{PAH} \mathrm{feature} \mathrm{(dashed} \mathrm{line),} \mathrm{with} \mathrm{the} \mathrm{shaded} \mathrm{area} \mathrm{indicating} \mathrm{the} \mathrm{spectral} \mathrm{range}$ used for obtaining the integrated flux. The red lines are the fitted local continua. For clarity, we applied a multiplicative factor to the IRS data (shown in the plot) for overlapped spectra. In a few cases, the nuclear data lie above the circumnuclear data; this can be due to calibration uncertainties.

(A color version of this figure is available in the online journal.) 

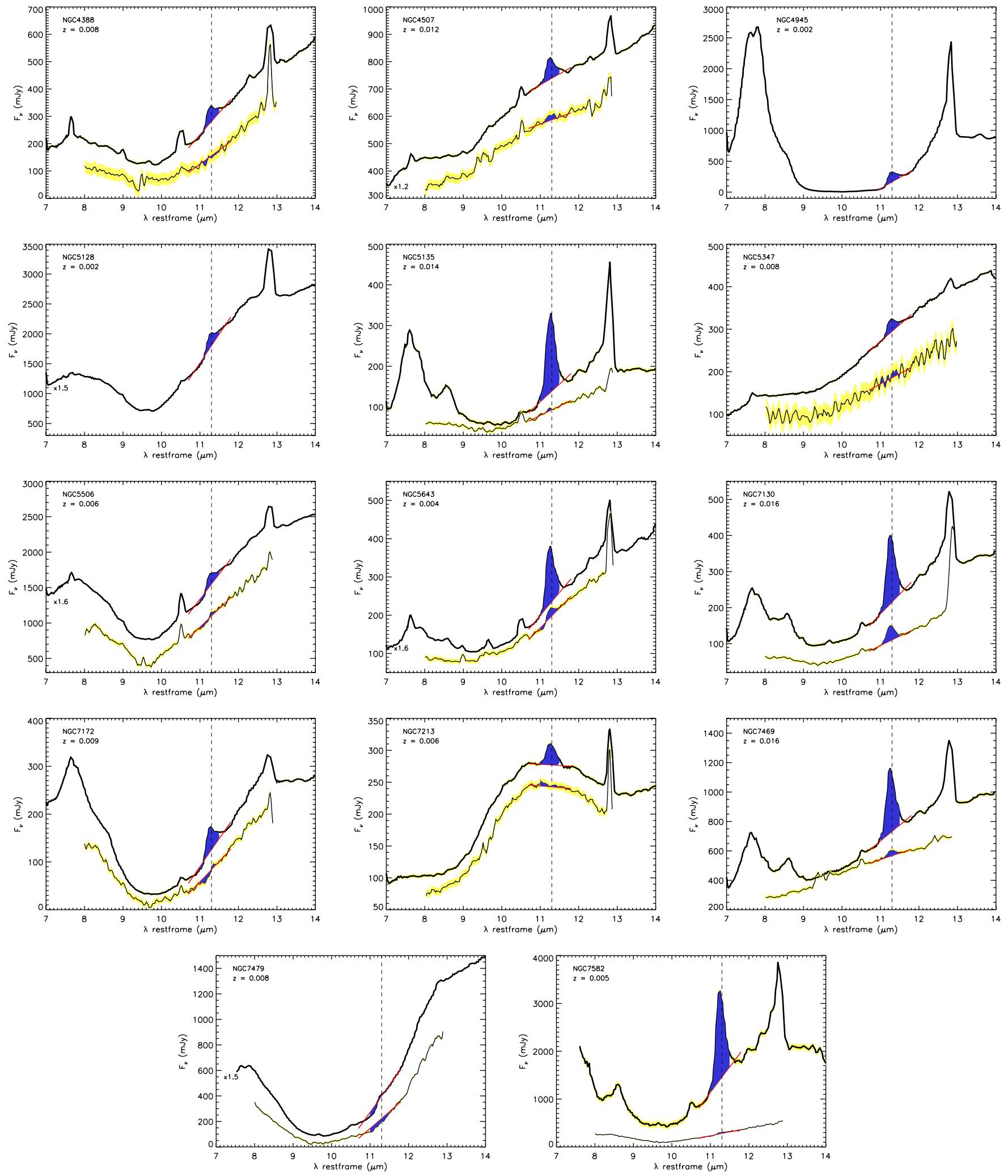

Figure 8. (Continued) 
agreement with the NSF on behalf of the Gemini partnership: the National Science Foundation (United States), the National Research Council (Canada), CONICYT (Chile), the Australian Research Council (Australia), Ministério da Ciência, Tecnologia e Inovação (Brazil), and Ministerio de Ciencia, Tecnología e Innovación Productiva (Argentina). The Cornell Atlas of Spitzer/ IRS Sources (CASSIS) is a product of the Infrared Science Center at Cornell University, supported by NASA and JPL.

\section{APPENDIX}

This Appendix includes the catalog of nuclear and circumnuclear spectra for the sample of galaxies.

\section{REFERENCES}

Akylas, A., Georgantopoulos, I., \& Comastri, A. 2001, MNRAS, 324, 521 Alexander, D. M., \& Hickox, R. C. 2012, NewAR, 56, 93

Alonso-Herrero, A., Ramos Almeida, C., Mason, R., et al. 2011, ApJ, 736,82

Alonso-Herrero, A., Sánchez-Portal, M., Ramos Almeida, C., et al. 2012, MNRAS, 425, 311

Antonucci, R. 1993, ARA\&A, 31, 473

Armus, L., Mazzarella, J. M., Evans, A. S., et al. 2009, PASP, 121, 559

Asmus, D., Gandhi, P., Smette, A., Hönig, S. F., \& Duschl, W. J. 2011, A\&A, 536, A36

Ballantyne, D. R. 2008, ApJ, 685, 787

Bassani, L., Dadina, M., Maiolino, R., et al. 1999, ApJS, 121, 473

Beckmann, V., Gehrels, N., Shrader, C. R., \& Soldi, S. 2006, ApJ, 638, 642

Beifiori, A., Sarzi, M., Corsini, E. M., et al. 2009, ApJ, 692, 856

Bianchi, S., Guainazzi, M., Matt, G., et al. 2005, A\&A, 442, 185

Bohlin, R. C., Savage, B. D., \& Drake, J. F. 1978, ApJ, 224, 132

Brightman, M., \& Nandra, K. 2011, MNRAS, 413, 1206

Cid Fernandes, R., Jr., \& Terlevich, R. 1995, MNRAS, 272, 423

Colina, L., Gonzalez Delgado, R., Mas-Hesse, J. M., \& Leitherer, C. 2002, ApJ, 579,545

Colling, M. 2011, PhD thesis, Univ. Oxford

Dadina, M. 2007, A\&A, 461, 1209

Davies, R. I., Müller Sánchez, F., Genzel, R., et al. 2007, ApJ, 671, 1388

Diamond-Stanic, A. M., \& Rieke, G. H. 2010, ApJ, 724, 140

Diamond-Stanic, A. M., \& Rieke, G. H. 2012, ApJ, 746, 168

Díaz-Santos, T., Alonso-Herrero, A., Colina, L., et al. 2008, ApJ, 685, 211

Díaz-Santos, T., Alonso-Herrero, A., Colina, L., et al. 2010, ApJ, 711, 328

Ferrarese, L., \& Merritt, D. 2000, ApJL, 539, L9

Galliano, F., Madden, S. C., Tielens, A. G. G. M., Peeters, E., \& Jones, A. P. 2008, ApJ, 679, 310

Gebhardt, K. 2000, ApJL, 539, L13

Ghisellini, G., Haardt, F., \& Matt, G. 1994, MNRAS, 267, 743

Glasse, A. C., Atad-Ettedgui, E. I., \& Harris, J. W. 1997, Proc. SPIE, 2871, 1197

González Delgado, R. M., Heckman, T., Leitherer, C., et al. 1998, ApJ, 505,174

González-Martín, O., Rodríguez-Espinosa, J. M., Díaz-Santos, T., et al. 2013, A\&A, 553, A35

Goulding, A. D., Alexander, D. M., Bauer, F. E., et al. 2012, ApJ, 755, 5

Guainazzi, M., Matt, G., Brandt, W., et al. 2000, A\&A, 356, 463

Guainazzi, M., Rodriguez, P., Fabian, A. C., Iwasawa, K., \& Matt, G. 2004, MNRAS, 355, 297

Heckman, T., Krolik, J., Meurer, G., et al. 1995, ApJ, 452, 549

Hernán-Caballero, A., \& Hatziminaoglou, E. 2011, MNRAS, 414, 500

Hicks, E. K. S., Davies, R. I., Maciejewski, W., et al. 2013, ApJ, 768, 107

Ho, L. C., Filippenko, A. V., \& Sargent, W. L. W. 1997, ApJS, 112, 315

Ho, L. C., \& Keto, E. 2007, ApJ, 658, 314

Hönig, S. F., Kishimoto, M., Gandhi, P., et al. 2010, A\&A, 515, A23

Hönig, S. F., Smette, A., Beckert, T., et al. 2008, A\&A, 485, L21

Hopkins, P. F. 2012, MNRAS, 420, L8

Hopkins, P. F., Hayward, C. C., Narayanan, D., \& Hernquist, L. 2012, MNRAS, 420, 320

Hopkins, P. F., \& Quataert, E. 2010, MNRAS, 407, 1529

Houck, J. R., Roellig, T. L., van Cleve, J., et al. 2004, ApJS, 154, 18

Huchra, J., \& Burg, R. 1992, ApJ, 393, 90

Imanishi, M. 2003, ApJ, 599, 918

Imanishi, M., \& Wada, K. 2004, ApJ, 617, 214

Kawakatu, N., \& Wada, K. 2008, ApJ, 681, 73
Kelly, B. C. 2007, ApJ, 665, 1489

King, A. R. 2010, MNRAS, 402, 1516

Kormendy, J., \& Ho, L. C. 2013, ARA\&A, 51, 511

Lagage, P. O., Pel, J. W., Authier, M., et al. 2004, Msngr, 117, 12

LaMassa, S. M., Heckman, T. M., Ptak, A., et al. 2012, ApJ, 758, 1

Lebouteiller, V., Barry, D. J., Spoon, H. W. W., et al. 2011, ApJS, 196, 8

Levenson, N. A., Radomski, J. T., Packham, C., et al. 2009, ApJ, 703, 390

Li, A., \& Draine, B. T. 2002, ApJ, 572, 232

Magorrian, J., Tremaine, S., Richstone, D., et al. 1998, AJ, 115, 2285

Maiolino, R., \& Rieke, G. H. 1995, ApJ, 454, 95

Malizia, A., Landi, R., Bassani, L., et al. 2007, ApJ, 668, 81

Marco, O., \& Brooks, K. J. 2003, A\&A, 398, 101

Marconi, A., Risaliti, G., Gilli, R., et al. 2004, MNRAS, 351, 169

Marinucci, A., Bianchi, S., Nicastro, F., Matt, G., \& Goulding, A. D. 2012, ApJ, 748, 130

Mason, R. E., Geballe, T., Packham, C., et al. 2006, ApJ, 640, 612

Mason, R. E., Levenson, N. A., Packham, C., et al. 2007, ApJ, 659, 241

Mason, R. E., Levenson, N. A., Shi, Y., et al. 2009, ApJL, 693, L136

Mason, R. E., Lopez-Rodriguez, E., Packham, C., et al. 2012, AJ, 144, 11

Meléndez, M., Kraemer, S. B., Schmitt, H. R., et al. 2008, ApJ, 689, 95

Miles, J. W., Houck, J. R., \& Hayward, T. L. 1994, ApJL, 425, L37

Mueller, M., Madejski, G. M., Done, C., \& Zycki, P. T. 2003, BAAS, 35,637

Mullaney, J. R., Alexander, D. M., Goulding, A. D., \& Hickox, R. C. 2011, MNRAS, 414, 1082

Nayakshin, S., \& Zubovas, K. 2012, MNRAS, 427, 372

Nenkova, M., Sirocky, M. M., Ivezić, Ž., \& Elitzur, M. 2008a, ApJ, 685,147

Nenkova, M., Sirocky, M. M., Nikutta, R., Ivezić, Ž., \& Elitzur, M. 2008b, ApJ, 685,160

Panessa, F., Bassani, L., Cappi, M., et al. 2006, A\&A, 455, 173

Peeters, E., Allamandola, L. J., Hudgins, D. M., Hony, S., \& Tielens, A. G. G. M. 2004, in ASP Conf. Ser. 309, Astrophysics of Dust, ed. A. N. Witt, G. C. Clayton, \& B. T. Draine (San Francisco, CA: ASP), 141

Peeters, E., Hony, S., Van Kerckhoven, C., et al. 2002, A\&A, 390, 1089

Pereira-Santaella, M., Diamond-Stanic, A. M., Alonso-Herrero, A., \& Rieke, G. H. 2010, ApJ, 725, 2270

Piconcelli, E., Bianchi, S., Guainazzi, M., Fiore, F., \& Chiaberge, M. 2007, A\&A, 466, 855

Ramos Almeida, C., Levenson, N. A., Alonso-Herrero, A., et al. 2011, ApJ, 731,92

Ramos Almeida, C., Levenson, N. A., Rodríguez Espinosa, J. M., et al. 2009, ApJ, 702, 1127

Ramos Almeida, C., Rodríguez Espinosa, J. M., Acosta-Pulido, J. A., et al. 2013, MNRAS, 429, 3449

Rieke, G. H., Alonso-Herrero, A., Weiner, B. J., et al. 2009, ApJ, 692, 556

Risaliti, G., Elvis, M., Fabbiano, G., Baldi, A., \& Zezas, A. 2005, ApJL, 623, L93

Roche, P. F., \& Aitken, D. K. 1985, MNRAS, 213, 789

Roche, P. F., Aitken, D. K., Smith, C. H., \& Ward, M. J. 1991, MNRAS, 248, 606

Roche, P. F., Packham, C., Aitken, D. K., \& Mason, R. E. 2007, MNRAS, 375,99

Roche, P. F., Packham, C., Telesco, C. M., et al. 2006, MNRAS, 367, 1689

Rush, B., Malkan, M. A., \& Spinoglio, L. 1993, ApJS, 89, 1

Sales, D. A., Pastoriza, M. G., Riffel, R., \& Winge, C. 2013, MNRAS, 429, 2634

Sales, D. A., Pastoriza, M. G., Riffel, R., et al. 2011, ApJ, 738, 109

Sandage, A., \& Tammann, G. A. 1987, A Revised Shapley-Ames Catalog of Bright Galaxies (2nd ed.; Washington, DC: Carnegie Institution of Washington)

Shi, Y., Ogle, P., Rieke, G. H., et al. 2007, ApJ, 669, 841

Siebenmorgen, R., Krügel, E., \& Spoon, H. W. W. 2004, A\&A, 414, 123

Silk, J., \& Rees, M. J. 1998, A\&A, 331, L1

Smith, J., Draine, B., Dale, D., et al. 2007, ApJ, 656, 770

Spoon, H. W. W., Tielens, A. G. G. M., Armus, L., et al. 2006, ApJ, 638,759

Storchi-Bergmann, T., Raimann, D., Bica, E. L. D., \& Fraquelli, H. A. 2000, ApJ, 544,747

Tacconi-Garman, L. E., \& Sturm, E. 2013, A\&A, 551, A139

Telesco, C. M., Ciardi, D., French, J., et al. 2003, Proc. SPIE, 4841, 913

Telesco, C. M., Pina, R. K., Hanna, K. T., et al. 1998, Proc. SPIE, 3354, 534

Thompson, T. A., Quataert, E., \& Murray, N. 2005, ApJ, 630, 167

Tielens, A. G. G. M. 2008, ARA\&A, 46, 289

Tielens, A. G. G. M. 2010, The Physics and Chemistry of the Interstellar Medium (Cambridge: Cambridge Univ. Press) 
Tueller, J., Mushotzky, R., Barthelmy, S., et al. 2008, ApJ, 681, 113

Uchida, K. I., Sellgren, K., Werner, M. W., \& Houdashelt, M. L. 2000, ApJ, 530,817

Voit, G. M. 1991, ApJ, 379, 122

Voit, G. M. 1992a, MNRAS, 258, 841

Voit, G. M. 1992b, in ASP Conf. Ser. 31, Relationships Between Active Galactic Nuclei and Starburst Galaxies, ed. A. V. Filippenko (San Francisco, CA: ASP), 87
Wada, K., \& Norman, C. A. 2002, ApJL, 566, L21

Wang, J., Fabbiano, G., Risaliti, G., et al. 2011, ApJ, 729, 75

Watabe, Y., Kawakatu, N., \& Imanishi, M. 2008, ApJ, 677, 895

Wild, V., Heckman, T., \& Charlot, S. 2010, MNRAS, 405, 933

Woo, J.-H., \& Urry, C. M. 2002, ApJ, 579, 530

Young, M., Elvis, M., \& Risaliti, G. 2010, ApJ, 708, 1388

Young, S., Packham, C., Mason, R. E., Radomski, J. T., \& Telesco, C. M. 2007, MNRAS, 378, 888 